\title{
Self-Restricted Green Fluorescent Protein Chromophore Analogs: Dramatic Emission Enhancement and Remarkable
}

\section{Solvatofluorochromism}

\author{
Hongping Deng, ${ }^{\dagger}$ Chunyang Yu, ${ }^{*, \dagger}$ Lidong Gong, ${ }^{\ddagger}$ and Xinyuan Zhu ${ }^{*}{ }^{\dagger}$ \\ † School of Chemistry and Chemical Engineering, State Key Laboratory of Metal Matrix \\ Composites, Shanghai Jiao Tong University, 800 Dongchuan Road, Shanghai 200240, P. R. \\ China. \\ $\$$ School of Chemistry and Chemical Engineering, Liaoning Normal University, 850 Huanghe \\ Road, Dalian 116029, P. R. China.
}

\section{Table of Contents}

$\begin{array}{ll}\text { 1. Experimental section } & \text { S2 }\end{array}$

Scheme S1, synthesis, characterizations and fluorescence measurements $\quad$ S2-S7

$\begin{array}{ll}\text { 2. Additional Data } & \text { S8 }\end{array}$

Figure S1 to Figure S5, Table S1 to Table S4 S8-S12

$\begin{array}{lr}\text { 3. Computational Details } & \text { S13 }\end{array}$

Figure S6 to Figure S8, Table S5 to Table S10 S13-S17

4. ${ }^{1} \mathrm{H}$ and ${ }^{13} \mathrm{C}$ NMR Spectra for GFPc Analogs $\quad \mathrm{S} 15$

Figure S9 to Figure S42 S18-S29

$\begin{array}{lr}\text { 5. IR spectra for GFPc Analogs } & \text { S27 }\end{array}$

Figure S43 to Figure S46 S30-S31

$\begin{array}{lr}\text { 6. References } & \text { S32 }\end{array}$ 


\section{Experimental section}

1.1 Synthesis and Characterizations. All reagents were purchased commercially and used directly unless otherwise mentioned. Solvents were dried and distilled according to standard methods. The compound methyl 2-(1-ethoxyethylideneamino)acetate (MEEA) was prepared as previously reported procedures. ${ }^{[1]}$ Varian MERCURY plus-400 spectrometer was applied to test the ${ }^{1} \mathrm{H}$ and ${ }^{13} \mathrm{C}$ nuclear magnetic resonance (NMR) spectra at $400 \mathrm{MHz}$ and $100 \mathrm{MHz}$ instruments respectively with proper deuterated solvent at $298 \mathrm{~K}$. Chemical shifts $(\delta)$ were measured in parts per million (ppm) and coupling constant $(\mathrm{J})$ was used in Hertz $(\mathrm{Hz})$. The residual peak of deuterated solvent conducted as reference to all chemical shifts. Perkin Elmer Paragon 1000 spectrophotometer was used to measure the Fourier transform infrared (FTIR) spectra between 4000 and $450 \mathrm{~cm}^{-1}$. All samples were tableted by pestling the solid sample with dry potassium bromide (KBr) and moulding under high pressure. High resolution mass spectrometer (HRMS) data were acquired on a Waters Micromass Q-TOF Premier mass spectrometer for each sample from 50 to $1000 \mathrm{Da}$ with a $0.10 \mathrm{~s}$ scan time and a $0.01 \mathrm{~s}$ interscan delay over a $10 \mathrm{~min}$ analysis time.

\section{Preparation of 2,5-dialkoxy phenylaldehyde}

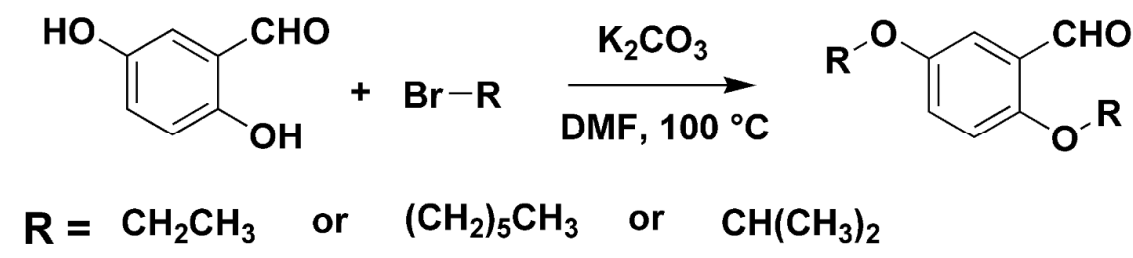

Scheme S1. General synthetic route of 2,5-dialkoxy phenylaldehyde.

1.1.1 General procedure for the synthesis of 2,5-dialkoxy phenylaldehydes. Overall, 2,5-dialkoxy phenylaldehydes were prepared as shown in Scheme $\mathrm{S} 1$. In a $100 \mathrm{~mL}$ reaction flask, anhydrous $\mathrm{K}_{2} \mathrm{CO}_{3}(30 \mathrm{mmol})$, 2,5-dihydroxybenzaldehyde $(10 \mathrm{mmol})$, and the corresponding bromoalkane (30 mmol) were stirred in DMF at $100{ }^{\circ} \mathrm{C}$ under nitrogen protection for $15 \mathrm{~h}$. After cooling to room temperature, DMF was removed under vacuum and DCM was added. The mixture was washed with water, dried with $\mathrm{MgSO}_{4}$, filtered and evaporated to get the crude product. Finally, the mixture was purified by silica gel column chromatography using ethyl acetate and petroleum ether as the solvents.

2,5-Diethoxybenzaldehyde. Yield 85\%. ${ }^{1} \mathrm{H}$ NMR (400 MHz, $\mathrm{CDCl}_{3}$, ppm, $298 \mathrm{~K}$ ): 10.47 (s, 1H), $7.31(\mathrm{~d}, J=3.2 \mathrm{~Hz}, 1 \mathrm{H}), 7.11(\mathrm{dd}, J=9.2 \mathrm{~Hz} J=3.2 \mathrm{~Hz}, 1 \mathrm{H}), 6.92(\mathrm{~d}, J=8.8 \mathrm{~Hz}, 1 \mathrm{H}), 4.11$ (q, $J$ $=6.8 \mathrm{~Hz}, 2 \mathrm{H}), 4.02(\mathrm{q}, J=6.8 \mathrm{~Hz}, 2 \mathrm{H}), 1.45(\mathrm{t}, J=6.8 \mathrm{~Hz}, 3 \mathrm{H}), 1.39(\mathrm{t}, J=6.8 \mathrm{~Hz}, 3 \mathrm{H}) \cdot{ }^{13} \mathrm{C}$ NMR (100 MHz, $\mathrm{CDCl}_{3}$, ppm, $\left.298 \mathrm{~K}\right)$ : 189.84, 156.22, 152.94, 125.20, 124.16, 114.56, 111.01, $64.91,64.18,14.87$.

2,5-Dihexyloxybenzaldehyde. Yield 81\%. ${ }^{1} \mathrm{H}$ NMR (400 MHz, $\mathrm{CDCl}_{3}, \mathrm{ppm}, 298 \mathrm{~K}$ ): 10.47 (s, $1 \mathrm{H}), 7.31(\mathrm{~d}, J=3.2 \mathrm{~Hz}, 1 \mathrm{H}), 7.11(\mathrm{dd}, J=9.2 \mathrm{~Hz} J=3.2 \mathrm{~Hz}, 1 \mathrm{H}), 6.92(\mathrm{~d}, J=9.2 \mathrm{~Hz}, 1 \mathrm{H}), 4.03$ (t, $J=6.4 \mathrm{~Hz}, 2 \mathrm{H}), 3.94$ (t, $J=6.4 \mathrm{~Hz}, 2 \mathrm{H}), 1.79(\mathrm{~m}, 4 \mathrm{H}), 1.45(\mathrm{~m}, 4 \mathrm{H}), 1.34(\mathrm{~m}, 8 \mathrm{H}), 0.89$ (m, $6 \mathrm{H}) .{ }^{13} \mathrm{C}$ NMR (100 MHz, CDCl 3 , ppm, $\left.298 \mathrm{~K}\right): 189.81,156.41,153.12,125.19,124.18,114.47$, $110.95,69.31,68.78,31.65,29.29,25.81,22.69,14.12$. 
2,5-Diisopropoxybenzaldehyde. Yield 63\%. ${ }^{1} \mathrm{H} \mathrm{NMR}\left(400 \mathrm{MHz}, \mathrm{CDCl}_{3}, \mathrm{ppm}, 298 \mathrm{~K}\right.$ ): 10.43 (s, $1 \mathrm{H}), 7.31(\mathrm{~d}, J=3.6 \mathrm{~Hz}, 1 \mathrm{H}), 7.08(\mathrm{dd}, J=9.2 \mathrm{~Hz}, J=3.2 \mathrm{~Hz}, 1 \mathrm{H}), 6.93(\mathrm{~d}, J=8.8 \mathrm{~Hz}, 1 \mathrm{H}), 4.56$ (dt, $J=6.4 \mathrm{~Hz}, 1 \mathrm{H}), 4.50$ (dt, $J=6.4 \mathrm{~Hz}, 1 \mathrm{H}), 1.37$ (s, 3H), 1.36 (s, 3H), $1.31(\mathrm{~s}, 3 \mathrm{H}), 1.30$ (s. 3H). ${ }^{13} \mathrm{C}$ NMR (100 MHz, $\mathrm{CDCl}_{3}$, ppm, $\left.298 \mathrm{~K}\right): 190.00,155.38,151.99,126.62,125.64,116.82$, $112.91,72.28,70.90,22.22,22.14$.

4.1.2 General procedure for the synthesis of GFPc analogs. GFPc analogs were conveniently synthesized through a 1,3-dipolar, [2+3] cycloaddition of an imide ylide with aromatic Schiff bases. ${ }^{35}$ Briefly, Schiff bases were prepared by combining the given aromatic aldehyde $(1 \mathrm{mmol})$ with propargylamine $(1.1 \mathrm{mmol})$ in ethanol for $12 \mathrm{~h}$ at room temperature. Then, the solvent was removed under vacuum and methyl 2-(1-ethoxyethylideneamino) acetate $(1.1 \mathrm{mmol})$ was added in ethanol. The reaction was allowed to stir overnight under ambient conditions. The progression of reactions was monitored by thin-layer-chromatography. Finally, the mixture was purified by silica gel column chromatography using ethyl acetate and petroleum ether as the solvents. Characterizations of synthetic chromophores are listed below.

4-(2-Methoxylbenzylidene)-1-propinyl-2-methyl-5-imidazolinone (2-MeOBDPI). Yellow solid, yield 75\%. ${ }^{1} \mathrm{H}$ NMR (400 MHz, $\mathrm{CDCl}_{3}$, ppm, $298 \mathrm{~K}$ ): 8.71 (dd, $\left.J=7.8 \mathrm{~Hz} J=1.4 \mathrm{~Hz}, 1 \mathrm{H}\right), 7.72$ (s, 1H), $7.35(\mathrm{~m}, 1 \mathrm{H}), 7.03(\mathrm{t}, J=7.6 \mathrm{~Hz}, 1 \mathrm{H}), 6.89(\mathrm{~d}, J=8.4 \mathrm{~Hz}, 1 \mathrm{H}), 4.41(\mathrm{~d}, J=2.4 \mathrm{~Hz}, 2 \mathrm{H}), 3.88$ (s, 3H), 2.50 (s, 3H), 2.29 (t, $J=2.6 \mathrm{~Hz}, 1 \mathrm{H}) .{ }^{13} \mathrm{C} \mathrm{NMR}\left(100 \mathrm{MHz}, \mathrm{CDCl}_{3}, \mathrm{ppm}, 298 \mathrm{~K}\right): 169.57$, $160.95,159.34,137.68,133.09,132.12,123.20,122.53$, 121.07, 110.85, 77.54, 72.74, 55.79, 29.57, 16.02. HRMS: $\mathrm{m} / \mathrm{z}$ calculated for $\left[\mathrm{C}_{15} \mathrm{H}_{15} \mathrm{~N}_{2} \mathrm{O}_{2}\right]^{+}: 255.1134$, found: 255.1137 . IR ( $\mathrm{KBr}$ ): $3225,3069,2967,2928,2848,2117,1718,1643,1596,1561,1486,1475,1441,1419,1403,1370$, $1338,1314,1251,1242,1207,1187,1160,1142,1103,1082,1049,1020,945,934,913,892,852$, $834,796,769,758,750,711,666,609,582,543,509,484,461 \mathrm{~cm}^{-1}$.

4-(3-Methoxylbenzylidene)-1-propinyl-2-methyl-5-imidazolinone (3-MeOBDPI). Yellow solid, yield 72\%. ${ }^{1} \mathrm{H}$ NMR (400 MHz, $\mathrm{CDCl}_{3}$, ppm, $298 \mathrm{~K}$ ): 7.81 (t, $\left.J=1.8 \mathrm{~Hz}, 1 \mathrm{H}\right), 7.64$ (d, $J=7.6 \mathrm{~Hz}$, $1 \mathrm{H}), 7.32(\mathrm{t}, J=8.0 \mathrm{~Hz}, 1 \mathrm{H}), 7.10(\mathrm{~s}, 1 \mathrm{H}), 6.94(\mathrm{dd}, J=8.2 \mathrm{~Hz} J=2.6 \mathrm{~Hz}, 1 \mathrm{H}), 4.40$ (d, $J=2.8$ $\mathrm{Hz}, 2 \mathrm{H}), 3.85$ (s, 3H), 2.49 (s, 3H), 2.30 (t, $J=2.4 \mathrm{~Hz}, 1 \mathrm{H}) .{ }^{13} \mathrm{C}$ NMR (100 MHz, $\mathrm{CDCl}_{3}, \mathrm{ppm}$, $298 \mathrm{~K}): 169.56,161.65,159.86,138.41,135.43,129.81,128.15,125.31,116.87,116.84,77.38$, 72.88, 55.50, 29.59, 16.06. HRMS: $\mathrm{m} / \mathrm{z}$ calculated for $\left[\mathrm{C}_{15} \mathrm{H}_{15} \mathrm{~N}_{2} \mathrm{O}_{2}\right]^{+}: 255.1134$, found: 255.1128 . IR (KBr): 3273, 3042, 2993, 2965, 2940, 2836, 2127, 1721, 1648, 1600, 1575, 1488, 1466, 1443, $1433,1407,1357,1341,1312,1270,1241,1193,1172,1160,1135,1080,1051,1031,1012,990$, $939,922,912,879,786,743,706,693,671,616,599,563,553,474 \mathrm{~cm}^{-1}$.

4-(4-Methoxylbenzylidene)-1-propinyl-2-methyl-5-imidazolinone (4-MeOBDPI). Yellow solid, yield, $81 \%$. ${ }^{1} \mathrm{H}$ NMR (400 MHz, $\mathrm{CDCl}_{3}$, ppm, $\left.298 \mathrm{~K}\right): 8.12$ (d, $\left.J=8.8 \mathrm{~Hz}, 2 \mathrm{H}\right), 7.12(\mathrm{~s}, 1 \mathrm{H}), 6.95$ (d, $J=9.4 \mathrm{~Hz}, 2 \mathrm{H}), 4.41$ (d, $J=2.4 \mathrm{~Hz}, 2 \mathrm{H}), 3.86(\mathrm{~s}, 3 \mathrm{H}), 2.50(\mathrm{~s}, 3 \mathrm{H}), 2.29$ (t, $J=2.4 \mathrm{~Hz}, 1 \mathrm{H})$. ${ }^{13} \mathrm{C}$ NMR (100 MHz, $\mathrm{CDCl}_{3}$, ppm, $298 \mathrm{~K}$ ): 169.58, 161.65, 160.23, 136.32, 134.42, 128.48, 127.15, 114.53, 77.50, 72.74, 55.58, 29.57, 15.97. HRMS: $\mathrm{m} / \mathrm{z}$ calculated for $\left[\mathrm{C}_{15} \mathrm{H}_{15} \mathrm{~N}_{2} \mathrm{O}_{2}\right]^{+}$: 255.1134, found: 255.1136. IR (KBr): 3241, 3073, 3001, 2959, 2935, 2853, 2839, 2123, 1716, 1701, 1642, 1596, 1571, 1507, 1465, 1439, 1423, 1404, 1367, 1341, 1319, 1309, 1251, 1217, 1175, 1138, 1113, 1083, 1032, 1009, 952, 938, 906, 893, 857, 847, 818, 780, 767, 752, 724, 712, 703, $665,629,616,603,542,491,475 \mathrm{~cm}^{-1}$.

4-(2,3-Dimethoxylbenzylidene)-1-propinyl-2-methyl-5-imidazolinone (2,3-MeOBDPI). Yield solid, yield 66\%. ${ }^{1} \mathrm{H}$ NMR (400 MHz, $\left.\mathrm{CDCl}_{3}, \mathrm{ppm}, 298 \mathrm{~K}\right): 8.32$ (dd, $J=8.0 \mathrm{~Hz} J=1.6 \mathrm{~Hz}, 1 \mathrm{H}$ ), 
$7.62(\mathrm{~s}, 1 \mathrm{H}), 7.12(\mathrm{t}, J=8.0 \mathrm{~Hz}, 1 \mathrm{H}), 6.95(\mathrm{dd}, J=8.2 \mathrm{~Hz} J=1.4 \mathrm{~Hz}, 1 \mathrm{H}), 4.41(\mathrm{~d}, J=2.4 \mathrm{~Hz}$, $2 \mathrm{H}), 3.88(\mathrm{~s}, 3 \mathrm{H}), 3.87(\mathrm{~s}, 3 \mathrm{H}), 2.50(\mathrm{~s}, 3 \mathrm{H}), 2.29(\mathrm{t}, J=2.6 \mathrm{~Hz}, 1 \mathrm{H}) .{ }^{13} \mathrm{C} \mathrm{NMR}\left(100 \mathrm{MHz}, \mathrm{CDCl}_{3}\right.$, ppm, $298 \mathrm{~K}): 169.51,161.59,152.79,149.85,138.70,128.34,124.51,124.36,122.29,114.62$, 77.43, 72.84, 61.99, 56.05, 29.58, 16.01. HRMS: $\mathrm{m} / \mathrm{z}$ calculated for $\left[\mathrm{C}_{16} \mathrm{H}_{17} \mathrm{~N}_{2} \mathrm{O}_{3}\right]^{+}: 285.1239$, found: 285.1242 . IR (KBr): 3236, 3005, 2964, 2938, 2834, 2119, 1712, 1646, 1581, 1564, 1481, 1444, 1432, 1397, 1357, 1329, 1313, 1299, 1280, 1240, 1183, 1176, 1136, 1092, 1077, 1030, 1009, $1001,994,963,939,912,881,793,782,745,712,699,667,631,608,594,574,535,498 \mathrm{~cm}^{-1}$.

4-(2,4-Dimethoxylbenzylidene)-1-propinyl-2-methyl-5-imidazolinone (2,4-MeOBDPI). Yellow solid, yield 70\%. ${ }^{1} \mathrm{H}$ NMR (400 MHz, $\mathrm{CDCl}_{3}, \mathrm{ppm}, 298 \mathrm{~K}$ ): 8.76 (d, J=8.8 Hz, 1H), $7.70(\mathrm{~s}, 1 \mathrm{H})$, $6.61(\mathrm{dd}, J=8.8 \mathrm{~Hz} J=2.4 \mathrm{~Hz}, 1 \mathrm{H}), 6.42(\mathrm{~d}, J=2.4 \mathrm{~Hz}, 1 \mathrm{H}), 4.43$ (d, $J=2.4 \mathrm{~Hz}, 2 \mathrm{H}), 3.87$ (s, $3 \mathrm{H}), 3.86(\mathrm{~s}, 3 \mathrm{H}), 2.55(\mathrm{~s}, 3 \mathrm{H}), 2.29$ (t, $J=2.4 \mathrm{~Hz}, 1 \mathrm{H}) .{ }^{13} \mathrm{C} \mathrm{NMR}\left(100 \mathrm{MHz}, \mathrm{CDCl}_{3}\right.$, ppm, $\left.298 \mathrm{~K}\right)$ : 169.56, 163.39, 161.03, 159.47, 135.72, 134.57, 122.67, 116.59, 106.11, 97.92, 77.70, 72.58, 55.80, 55.67, 29.51, 15.97. HRMS: $\mathrm{m} / \mathrm{z}$ calculated for $\left[\mathrm{C}_{16} \mathrm{H}_{17} \mathrm{~N}_{2} \mathrm{O}_{3}\right]^{+}: 285.1239$, found: 285.1252 . IR (KBr): 3221, 3009, 2979, 2926, 2853, 2116, 1708, 1634, 1615, 1572, 1564, 1504, 1467, 1451, 1437, 1422, 1400, 1365, 1333, 1311, 1295, 1279, 1246, 1208, 1180, 1162, 1149, 1113, 1084, 1044, $1035,937,925,888,828,798,773,760,730,709,663,639,628,610,586,532,517,504 \mathrm{~cm}^{-1}$.

4-(2,5-Dimethoxylbenzylidene)-1-propinyl-2-methyl-5-imidazolinone (2,5-MeOBDPI). Yellow solid, yield 61\%. ${ }^{1} \mathrm{H} \mathrm{NMR}\left(400 \mathrm{MHz}, \mathrm{CDCl}_{3}, \mathrm{ppm}, 298 \mathrm{~K}\right): 8.44$ (d, $\left.J=3.2 \mathrm{~Hz}, 1 \mathrm{H}\right), 7.70(\mathrm{~s}, 1 \mathrm{H})$, $6.95(\mathrm{dd}, J=9.2 \mathrm{~Hz}, J=3.2 \mathrm{~Hz}, 1 \mathrm{H}), 6.85(\mathrm{~d}, J=9.2 \mathrm{~Hz}, 1 \mathrm{H}), 4.43$ (d, $J=2.4 \mathrm{~Hz}, 2 \mathrm{H}), 3.87$ (s, $3 \mathrm{H}), 3.86(\mathrm{~s}, 3 \mathrm{H}), 2.52(\mathrm{~s}, 3 \mathrm{H}), 2.31(\mathrm{t}, J=2.6 \mathrm{~Hz}, 1 \mathrm{H}) .{ }^{13} \mathrm{C} \mathrm{NMR}\left(100 \mathrm{MHz}, \mathrm{CDCl}_{3}\right.$, ppm, $\left.298 \mathrm{~K}\right)$ : 169.54, 160.94, 154.19, 153.67, 137.86, 123.75, 122.24, 118.49, 117.18, 112.08, 77.56, 72.75, 56.42, 56.00, 29.57, 16.11. HRMS: $\mathrm{m} / \mathrm{z}$ calculated for $\left[\mathrm{C}_{16} \mathrm{H}_{17} \mathrm{~N}_{2} \mathrm{O}_{3}\right]^{+}: 285.1239$, found: 285.1241 . IR (KBr): 3250, 3221, 3083, 2989, 2953, 2933, 2905, 2825, 2116, 1712, 1644, 1578, 1568, 1493, 1460, 1437, 1426, 1418, 1401, 1367, 1341, 1320, 1304, 1285, 1260, 1241, 1222, 1194, 1172, 1145, $1085,1058,1023,942,929,920,907,889,808,800,769,739,709,685,664,614,563,546,533$, $519,491 \mathrm{~cm}^{-1}$.

4-(3,4-Dimethoxylbenzylidene)-1-propinyl-2-methyl-5-imidazolinone (3,4-MeOBDPI). Yellow solid, yield 63\%. ${ }^{1} \mathrm{H}$ NMR (400 MHz, $\mathrm{CDCl}_{3}$, ppm, $298 \mathrm{~K}$ ): 8.03 (d, $\left.J=2.0 \mathrm{~Hz}, 1 \mathrm{H}\right), 7.56$ (d, $J=$ $8.4 \mathrm{~Hz}, 1 \mathrm{H}), 7.10$ (s, 1H), 6.90 (d, $J=8.4 \mathrm{~Hz}, 1 \mathrm{H}), 4.41$ (d, $J=2.4 \mathrm{~Hz}, 2 \mathrm{H}), 3.96(\mathrm{~s}, 3 \mathrm{H}), 3.93$ (s, 3H), 2.51 (s, 3H), 2.29 (t, $J=2.6 \mathrm{~Hz}, 1 \mathrm{H}) .{ }^{13} \mathrm{C} \mathrm{NMR}\left(100 \mathrm{MHz}, \mathrm{CDCl}_{3}\right.$, ppm, $\left.298 \mathrm{~K}\right): 169.57$, 160.26, 151.49, 149.24, 136.50, 128.61, 127.50, 127.31, 114.39, 111.08, 77.54, 72.78, 56.17, 56.14, 29.61, 16.13. HRMS: $\mathrm{m} / \mathrm{z}$ calculated for $\left[\mathrm{C}_{16} \mathrm{H}_{17} \mathrm{~N}_{2} \mathrm{O}_{3}\right]^{+}: 285.1239$, found: 285.1235 . IR (KBr): 3217, 3009, 2958, 2922, 2849, 2835, 2116, 1705, 1643, 1595, 1579, 1515, 1471, 1454, 1442, 1427, 1400, 1368, 1334, 1307, 1275, 1249, 1207, 1173, 1151, 1139, 1083, 1043, 1032, 1015, $953,935,915,899,874,818,810,781,768,763,719,638,610,593,547,482 \mathrm{~cm}^{-1}$.

4-(3,5-Dimethoxylbenzylidene)-1-propinyl-2-methyl-5-imidazolinone (3,5-MeOBDPI).Yellow solid, yield 72\%. ${ }^{1} \mathrm{H}$ NMR (400 MHz, $\mathrm{CDCl}_{3}, \mathrm{ppm}, 298 \mathrm{~K}$ ): $7.34(\mathrm{~d}, J=2.0 \mathrm{~Hz}, 2 \mathrm{H}), 7.08(\mathrm{~s}, 1 \mathrm{H})$, 6.53 (t, $J=2.2 \mathrm{~Hz}, 1 \mathrm{H}), 4.41(\mathrm{~d}, J=2.4 \mathrm{~Hz}, 2 \mathrm{H}), 3.84(\mathrm{~s}, 6 \mathrm{H}), 2.54(\mathrm{~s}, 3 \mathrm{H}), 2.31$ (t, $J=2.6 \mathrm{~Hz}$, 1H). ${ }^{13} \mathrm{C}$ NMR (100 MHz, $\mathrm{CDCl}_{3}$, ppm, $\left.298 \mathrm{~K}\right): 169.61,161.72,160.96,138.58,135.85,128.28$, $110.21,103.44,77.39,72.90,55.68,29.64,16.15$. HRMS: $\mathrm{m} / \mathrm{z}$ calculated for $\left[\mathrm{C}_{16} \mathrm{H}_{17} \mathrm{~N}_{2} \mathrm{O}_{3}\right]^{+}$: 285.1239, found: 285.1233. IR (KBr): 3218, 3106, 3010, 2993, 2964, 2935, 2852, 2837, 2115, 1712, 1651, 1594, 1568, 1464, 1441, 1427, 1402, 1366, 1349, 1330, 1313, 1293, 1264, 1233, 1208 , $1159,1139,1089,1063,1017,950,930,916,884,860,826,786,765,729,676,657,622,616$, $592,544 \mathrm{~cm}^{-1}$. 
4-(2,3,4-Trimethoxylbenzylidene)-1-propinyl-2-methyl-5-imidazolinone $\quad(2,3,4-M e O B D P I)$. Yellow solid, yield 53\%. ${ }^{1} \mathrm{H}$ NMR (400 MHz, $\mathrm{CDCl}_{3}$, ppm, $298 \mathrm{~K}$ ): 8.53 (d, $J=8.8 \mathrm{~Hz}, 1 \mathrm{H}$ ), 7.54 (s, 1H), 6.77 (d, $J=9.2 \mathrm{~Hz}, 1 \mathrm{H}), 4.41$ (d, $J=2.4 \mathrm{~Hz}, 2 \mathrm{H}), 3.94(\mathrm{~s}, 3 \mathrm{H}), 3.91(\mathrm{~s}, 3 \mathrm{H}), 3.97$ (s, 3H), 2.49 (s, 3H), $2.29(\mathrm{t}, J=2.4 \mathrm{~Hz}, 1 \mathrm{H}) .{ }^{13} \mathrm{C}$ NMR $\left(100 \mathrm{MHz}, \mathrm{CDCl}_{3}, \mathrm{ppm}, 298 \mathrm{~K}\right): 169.49,160.37$, 156.12, 154.64, 142.11, 136.95, 128.57, 122.59, 121.39, 107.99, 77.59, 72.73, 62.18, 61.13, 56.30, 29.57, 16.02. HRMS: $\mathrm{m} / \mathrm{z}$ calculated for $\left[\mathrm{C}_{17} \mathrm{H}_{19} \mathrm{~N}_{2} \mathrm{O}_{4}\right]^{+}: 315.1345$, found: 315.1351 . IR ( $\mathrm{KBr}$ ): 3260, 2996, 2970, 2945, 2841, 2123, 1700, 1648, 1593, 1562, 1497, 1465, 1439, 1417, 1404, 1359, 1341, 1307, 1286, 1243, 1204, 1172, 1139, 1091, 1053, 1029, 1006, 945, 932, 916, 900, 878, 805, $796,763,751,716,699,682,643,631,609,599,509 \mathrm{~cm}^{-1}$.

4-(2,4,5-Trimethoxylbenzylidene)-1-propinyl-2-methyl-5-imidazolinone (2,4,5-MeOBDPI). Yellow solid, yield 55\%. ${ }^{1} \mathrm{H}$ NMR (400 MHz, $\mathrm{CDCl}_{3}$, ppm, $298 \mathrm{~K}$ ): 8.53 (s, 1H), $7.68(\mathrm{~s}, 1 \mathrm{H})$, $6.47(\mathrm{~s}, 1 \mathrm{H}), 4.41(\mathrm{~d}, J=2.4 \mathrm{~Hz}, 2 \mathrm{H}), 3.95(\mathrm{~s}, 3 \mathrm{H}), 3.94(\mathrm{~s}, 3 \mathrm{H}), 3.88(\mathrm{~s}, 3 \mathrm{H}), 2.50(\mathrm{~s}, 3 \mathrm{H}), 2.28(\mathrm{t}$, $J=2.6 \mathrm{~Hz}, 1 \mathrm{H}) .{ }^{13} \mathrm{C} \mathrm{NMR}\left(100 \mathrm{MHz}, \mathrm{CDCl}_{3}, \mathrm{ppm}, 298 \mathrm{~K}\right): 169.58,159.20,155.83,152.86$, 143.51, 135.59, 122.50, 115.37, 115.07, 96.22, 77.74, 72.60, 56.68, 56.53, 56.20, 29.55, 16.13 . HRMS: $\mathrm{m} / \mathrm{z}$ calculated for $\left[\mathrm{C}_{17} \mathrm{H}_{19} \mathrm{~N}_{2} \mathrm{O}_{4}\right]^{+}: 315.1345$, found: 315.1348 . IR $(\mathrm{KBr}): 3215,3006$, 2924, 2835, 2115, 1702, 1640, 1607, 1575, 1560, 1520, 1505, 1473, 1436, 1412, 1402, 1369, 1331, 1303, 1288, 1244, 1220, 1211, 1185, 1149, 1126, 1083, 1045, 1026, 1013, 935, 917, 899, 882, 814, $781,765,730,695,655,621,529 \mathrm{~cm}^{-1}$.

4-(3,4,5-Trimethoxylbenzylidene)-1-propinyl-2-methyl-5-imidazolinone (3,4,5-MeOBDPI). Yellow solid, yield 59\%. ${ }^{1} \mathrm{H}$ NMR (400 MHz, $\left.\mathrm{CDCl}_{3}, \mathrm{ppm}, 298 \mathrm{~K}\right): 7.46(\mathrm{~s}, 2 \mathrm{H}), 7.06(\mathrm{~s}, 1 \mathrm{H})$, $4.41(\mathrm{~d}, J=2.8 \mathrm{~Hz}, 2 \mathrm{H}), 3.92(\mathrm{~s}, 6 \mathrm{H}), 3.91(\mathrm{~s}, 3 \mathrm{H}), 2.53(\mathrm{~s}, 3 \mathrm{H}), 2.30(\mathrm{t}, J=2.6 \mathrm{~Hz}, 1 \mathrm{H}) .{ }^{13} \mathrm{C}$ NMR (100 MHz, $\mathrm{CDCl}_{3}$, ppm, $298 \mathrm{~K}$ ): 169.55, 161.16, 153.37, 140.57, 137.57, 129.71, 128.32, 109.85, 77.44, 72.87, 61.23, 56.39, 29.64, 16.19. HRMS: $\mathrm{m} / \mathrm{z}$ calculated for $\left[\mathrm{C}_{17} \mathrm{H}_{19} \mathrm{~N}_{2} \mathrm{O}_{4}\right]^{+}$: 315.1345, found: 315.1342. IR (KBr): 3236, 3105, 3034, 3009, 2960, 2932, 2838, 2119, 1709, 1646, 1581, 1505, 1464, 1457, 1436, 1424, 1409, 1368, 1331, 1321, 1251, 1233, 1184, 1164, 1142, 1130, 1085, 1052, 1021, 1008, 995, 964, 939, 887, 866, 852, 792, 780, 766, 751, 726, 681, 663, $626,615,529,518 \mathrm{~cm}^{-1}$.

4-(2,5-Difluorobenzylidene)-1-propinyl-2-methyl-5-imidazolinone (2,5-FBDPI). Light yellow solid, yield 21\%. ${ }^{1} \mathrm{H}$ NMR (400 MHz, DMSO- $\left.d_{6}, 298 \mathrm{~K}\right): 8.52(\mathrm{~m}, 1 \mathrm{H}), 7.37(\mathrm{~m}, 2 \mathrm{H}), 6.98(\mathrm{~s}, 1 \mathrm{H})$, 4.47 (d, $J=2.8 \mathrm{~Hz}, 2 \mathrm{H}), 3.40$ (t, $J=2.6 \mathrm{~Hz}, 1 \mathrm{H}), 2.47$ (s, 3H). ${ }^{13} \mathrm{C} \mathrm{NMR}\left(100 \mathrm{MHz}, \mathrm{CDCl}_{3}, \mathrm{ppm}\right.$, $298 \mathrm{~K}): 169.04,163.20,159.71$ (d, $J=396 \mathrm{~Hz}), 157.25$ (d, $J=487 \mathrm{~Hz}), 139.96,123.64,118.89$ (d, $J=264 \mathrm{~Hz}), 118.53(\mathrm{dd}, J=249 \mathrm{~Hz} J=85 \mathrm{~Hz}), 117.56,116.53(\mathrm{dd}, J=249 \mathrm{~Hz} J=85 \mathrm{~Hz}), 77.13$, 73.05, 29.67, 16.11. HRMS: $\mathrm{m} / \mathrm{z}$ calculated for $\left[\mathrm{C}_{14} \mathrm{H}_{11} \mathrm{~N}_{2} \mathrm{OF}_{2}\right]^{+}: 261.0839$, found: 261.0848 . IR (KBr): 3314, 2955, 2929, 2857, 2126, 1718, 1640, 1573, 1565, 1497, 1468, 1429, 1404, 1385, 1340, 1314, 1288, 1259, 1240, 1222, 1180, 1142, 1083, 1033, 937, 893, 807, 770, 723, 712, 471, $664,637,612,554 \mathrm{~cm}^{-1}$.

4-(2,5-Dichlorobenzylidene)-1-propinyl-2-methyl-5-imidazolinone (2,5-ClBDPI). Light yellow solid, yield 15\%. ${ }^{1} \mathrm{H}$ NMR (400 MHz, DMSO- $\left.d_{6}, 298 \mathrm{~K}\right): 8.83(\mathrm{~d}, J=2.8 \mathrm{~Hz}, 1 \mathrm{H}), 7.61$ (d, $J=8.4$ $\mathrm{Hz}, 1 \mathrm{H}), 7.51(\mathrm{dd}, J=8.6 \mathrm{~Hz} J=2.6 \mathrm{~Hz}, 1 \mathrm{H}), 7.14(\mathrm{~s}, 1 \mathrm{H}), 4.47$ (d, $J=2.4 \mathrm{~Hz}, 2 \mathrm{H}), 3.41$ (t, $J=$ $2.6 \mathrm{~Hz}, 1 \mathrm{H}), 2.47$ (s, 3H). ${ }^{13} \mathrm{C} \mathrm{NMR}\left(100 \mathrm{MHz}, \mathrm{CDCl}_{3}, \mathrm{ppm}, 298 \mathrm{~K}\right): 169.11,163.72,140.21$, 134.59, 133.49, 133.08, 131.15, 130.89, 130.86, 121.42, 77.11, 73.07, 29.70, 16.12. HRMS: m/z calculated for $\left[\mathrm{C}_{14} \mathrm{H}_{11} \mathrm{~N}_{2} \mathrm{OCl}_{2}\right]^{+}: 293.0248$, found: 293.0256. IR (KBr): 3254, 3067, 2960, 2923, $2852,2124,1707,1645,1580,1562,1460,1451,1439,1397,1364,1340,1313,1269,1241,1142$, $1124,1097,1045,1010,941,909,901,808,780,767,726,709,691,660,614,582,518 \mathrm{~cm}^{-1}$. 
4-(2,5-Dimethylbenzylidene)-1-propinyl-2-methyl-5-imidazolinone (2,5-MeBDPI). Light yellow solid, yield 73\%. ${ }^{1} \mathrm{H}$ NMR (400 MHz, DMSO- $\left.d_{6}, 298 \mathrm{~K}\right): 8.42(\mathrm{~s}, 1 \mathrm{H}), 7.15(\mathrm{~d}, J=7.6 \mathrm{~Hz}, 1 \mathrm{H})$, $7.12(\mathrm{~s}, 1 \mathrm{H}), 7.11(\mathrm{dd}, J=8.0 \mathrm{~Hz} J=1.6 \mathrm{~Hz}, 1 \mathrm{H}), 4.45(\mathrm{~d}, J=2.4 \mathrm{~Hz}, 2 \mathrm{H}), 3.38$ (t, $J=2.4 \mathrm{~Hz}$, 1H), 2.43 (s, 3H), 2.37 (s, 3H), 2.28 (s, 3H). ${ }^{13} \mathrm{C} \mathrm{NMR} \mathrm{(100} \mathrm{MHz,} \mathrm{CDCl}_{3}$, ppm, $298 \mathrm{~K}$ ): 169.42, 161.39, 137.73, 136.62, 135.80, 132.38, 132.24, 131.28, 130.41, 125.57, 77.22, 72.63, 29.41, 21.18, 19.67, 15.85. HRMS: $\mathrm{m} / \mathrm{z}$ calculated for $\left[\mathrm{C}_{16} \mathrm{H}_{17} \mathrm{~N}_{2} \mathrm{O}\right]^{+}: 253.1341$, found: 253.1329 . IR (KBr): 3199, 2963, 2920, 2853, 2120, 1710, 1637, 1605, 1555, 1497, 1443, 1425, 1408, 1381, 1374, 1350, 1324, 1297, 1287, 1258, 1235, 1209, 1170, 1145, 1110, 1089, 1033, 1007, 973, 943, $930,914,892,831,798,769,715,699,666,614,559,492 \mathrm{~cm}^{-1}$.

4-(2,5-Diethoxybenzylidene)-1-propinyl-2-methyl-5-imidazolinone (2,5-EtOBDPI). Yellow solid, yield 68\%. ${ }^{1} \mathrm{H}$ NMR (400 MHz, DMSO- $\left.d_{6}, 298 \mathrm{~K}\right): 8.33$ (t, $\left.J=1.6 \mathrm{~Hz}, 1 \mathrm{H}\right), 7.32(\mathrm{~s}, 1 \mathrm{H}), 6.98$ (d, $J=2.0 \mathrm{~Hz}, 2 \mathrm{H}), 4.44$ (d, $J=2.4 \mathrm{~Hz}, 2 \mathrm{H}), 4.06$ (q, $J=6.8 \mathrm{~Hz}, 2 \mathrm{H}), 3.97$ (q, $J=6.8 \mathrm{~Hz}, 2 \mathrm{H}), 3.37$ (t, $J=2.4 \mathrm{~Hz}, 1 \mathrm{H}), 2.43(\mathrm{~s}, 3 \mathrm{H}), 1.33(\mathrm{dt}, J=2.4 \mathrm{~Hz}, 6 \mathrm{H}) \cdot{ }^{13} \mathrm{C} \mathrm{NMR}\left(100 \mathrm{MHz}, \mathrm{CDCl}_{3}, \mathrm{ppm}, 298 \mathrm{~K}\right)$ : $169.32,160.51,153.32,152.72,137.45,123.73,122.52$, 119.06, 117.55, 113.07, 77.31, 72.50, 64.83, 63.97, 29.34, 21.18, 15.88, 14.94. HRMS: $\mathrm{m} / \mathrm{z}$ calculated for $\left[\mathrm{C}_{18} \mathrm{H}_{21} \mathrm{~N}_{2} \mathrm{O}_{3}\right]^{+}: 313.1552$, found: 313.1554. IR (KBr): 3268, 3078, 2980, 2935, 2877, 2126, 1712, 1639, 1575, 1560, 1497, 1471, 1435, 1420, 1401, 1366, 1336, 1312, 1293, 1241, 1214, 1185, 1145, 1109, 1084, 1052, 1041 , $969,925,893,802,773,764,751,710,670,612,550 \mathrm{~cm}^{-1}$.

4-(2,5-Dihexyloxybenzylidene)-1-propinyl-2-methyl-5-imidazolinone (2,5-HexOBDPI). Yellow solid, yield 57\%. ${ }^{1} \mathrm{H}$ NMR (400 MHz, DMSO- $\left.d_{6}, 298 \mathrm{~K}\right): 8.32$ (t, $\left.J=1.6 \mathrm{~Hz}, 1 \mathrm{H}\right), 7.33(\mathrm{~s}, 1 \mathrm{H})$, $6.98(\mathrm{~d}, J=1.6 \mathrm{~Hz}, 2 \mathrm{H}), 4.43(\mathrm{~d}, J=2.4 \mathrm{~Hz}, 2 \mathrm{H}), 3.99$ (t, $J=2.4 \mathrm{~Hz}, 2 \mathrm{H}), 3.91(\mathrm{t}, J=2.4 \mathrm{~Hz}, 2 \mathrm{H})$, $3.37(\mathrm{t}, J=2.4 \mathrm{~Hz}, 1 \mathrm{H}), 2.42(\mathrm{~s}, 3 \mathrm{H}), 1.69(\mathrm{~m}, 4 \mathrm{H}), 1.41(\mathrm{~m}, 4 \mathrm{H}), 1.29(\mathrm{~m}, 8 \mathrm{H}), 0.86(\mathrm{~m}, 6 \mathrm{H}) .{ }^{13} \mathrm{C}$ NMR (100 MHz, $\mathrm{CDCl}_{3}$, ppm, $\left.298 \mathrm{~K}\right): 169.30,160.45,153.48,152.90,137.38,123.72,122.54$, $119.13,117.49,113.09,77.33,72.47,69.41,68.59,31.66,31.58,29.34,29.32,29.24,25.79,25.77$, 22.66, 22.60, 15.89, 14.08, 14.06. HRMS: $\mathrm{m} / \mathrm{z}$ calculated for $\left[\mathrm{C}_{26} \mathrm{H}_{37} \mathrm{~N}_{2} \mathrm{O}_{3}\right]^{+}: 425.2804$, found: 425.2796. IR (KBr): 3314, 2955, 2929, 2857, 2126, 1718, 1640, 565, 1497, 1468, 1429, 1404 , $1340,1314,1288,1240,1222,1180,1142,1083,1033,937,893,807,770,712,664,612,554$ $\mathrm{cm}^{-1}$.

4-(2,5-Diisopropoxybenzylidene)-1-propinyl-2-methyl-5-imidazolinone (2,5-IprOBDPI). Yellow solid, yield 51\%. ${ }^{1} \mathrm{H} \mathrm{NMR}\left(400 \mathrm{MHz}, \mathrm{CDCl}_{3}, \mathrm{ppm}, 298 \mathrm{~K}\right): 8.40$ (d, $\left.J=2.8 \mathrm{~Hz}, 1 \mathrm{H}\right), 7.67(\mathrm{~s}, 1 \mathrm{H})$, $6.89(\mathrm{dd}, J=8.8 \mathrm{~Hz} J=2.8 \mathrm{~Hz}, 1 \mathrm{H}), 6.82(\mathrm{~d}, J=8.8 \mathrm{~Hz}, 1 \mathrm{H}), 4.50(\mathrm{~m}, 2 \mathrm{H}), 4.40(\mathrm{~d}, J=2.8 \mathrm{~Hz}$, 2H), 2.49 (s, 3H), 2.28 (t, $J=2.4 \mathrm{~Hz}, 1 \mathrm{H}), 1.35$ (d, $J=3.6 \mathrm{~Hz}, 6 \mathrm{H}), 1.34(\mathrm{~d}, J=3.6 \mathrm{~Hz}, 6 \mathrm{H}) .{ }^{13} \mathrm{C}$ NMR (100 MHz, $\mathrm{CDCl}_{3}$, ppm, $\left.298 \mathrm{~K}\right): 169.58,160.55,152.61$, 151.91, 137.63, 125.30, 123.07, $120.89,119.38,115.75,77.57,72.64,72.33,70.85,29.54,22.34,16.08$. HRMS: $\mathrm{m} / \mathrm{z}$ calculated for $\left[\mathrm{C}_{20} \mathrm{H}_{25} \mathrm{~N}_{2} \mathrm{O}_{3}\right]^{+}:$341.1865, found: 341.1871. IR (KBr): 3241, 3097, 2965, 2932, 2122, 1720, 1632, 1576, 1559, 1496, 1467, 1445, 1427, 1407, 1393, 1370, 1333, 1312, 1302, 1288, 1260, 1244, 1228, 1189, 1142, 1118, 1103, 1081, 1016, 979, 958, 941, 922, 884, 859, 810, 770, 762, 722, 664, 615, $550 \mathrm{~cm}^{-1}$.

1.2 Absorption and Fluorescence Spectroscopy. Perkin Elmer Lambda 20 UV-Vis spectrometer was utilized to test the ultraviolet-visible (UV-Vis) absorption spectra of sample solutions at room temperature with a scan speed of $480 \mathrm{~nm} / \mathrm{min}$. The steady-state fluorescence spectra were tested on a PTI-QM/TM/IM steady-state \& time-resolved fluorescence spectrofluorometer (USA/CAN Photon Technology International Int.). Excitation wavelength was the maximum UV-Vis absorption wavelength. Fluorescence quantum yields were determined 
using quinine sulfate ( $\Phi=0.54$ in $1.0 \mathrm{~N}$ sulfuric acid) as the standard and calculated using the formula below: ${ }^{[2]}$

$$
\Phi_{\mathrm{fl}}^{\text {sample }}=\Phi_{\mathrm{fl}}^{\text {standard }} \times\left(\mathrm{F}^{\text {sample }} / \mathrm{F}^{\text {standard }}\right) \times\left(\mathrm{n}^{\text {sample }} / \mathrm{n}^{\text {standard }}\right)^{2} \times\left(\mathrm{A}^{\text {standard }} / \mathrm{A}^{\text {sample }}\right)
$$

Where $\Phi$ is the fluorescence quantum yield; $F$ denotes the area under the corrected emission spectra; $A$ is the absorbance at the excitation wavelength; $n$ denotes the refractive index of the solvent.

1.3 Fluorescence Lifetime. The time-resolved fluorescence spectra were recorded on a PTI-QM/TM/IM steady-state \& time-resolved fluorescence spectrofluorometer (USA/CAN Photon technology international Int.) by using the time-correlated single-photon counting (TCSPC) technique. Utilizing the picosecond pulses from a doubled frequency, the time-resolved fluorescence spectra of sample solutions were excited with maximum UV-Vis absorption wavelength and detected at the maximum emission wavelength for lifetime measurements with an emission polarizer and depolarizer. 


\section{Additional Data}

Table S1. Absorption $\left(\lambda_{\text {abs }}\right)$ and fluorescence $\left(\lambda_{\mathrm{f}}\right)$ maxima (in $\mathrm{nm}$ ) and fluorescence quantum yields $\left(\Phi_{\mathrm{f}}\right)$ of methoxyl-substituted GFPc analogs in EA.

\begin{tabular}{|c|c|c|c|c|c|c|c|}
\hline Sample & $\lambda_{\text {abs }}$ & $\lambda_{\mathrm{f}}$ & $\Phi_{\mathrm{f}}(\%)$ & Sample & $\lambda_{\mathrm{abs}}$ & $\lambda_{\mathrm{f}}$ & $\Phi_{\mathrm{f}}(\%)$ \\
\hline 2-MeOBDPI & 371 & 428 & 0.2 & $3,4-\mathrm{MeOBDPI}$ & 373 & 434 & 0.1 \\
\hline 3-MeOBDPI & 358 & 424 & 0.2 & $3,5-\mathrm{MeOBDPI}$ & 356 & 454 & 1.4 \\
\hline 4-MeOBDPI & 366 & 432 & 0.1 & $2,3,4-\mathrm{MeOBDPI}$ & 371 & 433 & 0.1 \\
\hline 2,3-MeOBDPI & 358 & 454 & 0.7 & $2,4,5-\mathrm{MeOBDPI}$ & 403 & 465 & 0.1 \\
\hline 2,4-MeOBDPI & 381 & 434 & 0.1 & $3,4,5-\mathrm{MeOBDPI}$ & 368 & 444 & 0.9 \\
\hline 2,5-MeOBDPI & 393 & 475 & 10.1 & & & & \\
\hline
\end{tabular}

Table S2. Absorption $\left(\lambda_{\text {abs }}\right)$, fluorescence $\left(\lambda_{\mathrm{f}}\right)$ maxima (in $\mathrm{nm}$ ), fluorescence quantum yields $\left(\Phi_{\mathrm{f}}\right)$ of 2,5-disubstituted chromophores in EA.

\begin{tabular}{|c|c|c|c|c|c|c|c|}
\hline Sample & $\lambda_{\text {abs }}$ & $\lambda_{\mathrm{f}}$ & $\Phi_{\mathrm{f}}(\%)$ & Sample & $\lambda_{\mathrm{abs}}$ & $\lambda_{\mathrm{f}}$ & $\Phi_{\mathrm{f}}(\%)$ \\
\hline 2,5-FBDPI & 356 & 423 & 2.1 & 2,5 -EtOBDPI & 394 & 483 & 11.1 \\
\hline 2,5-ClBDPI & 359 & 428 & 3.4 & 2,5 -HexOBDPI & 397 & 484 & 11.4 \\
\hline 2,5-MeBDPI & 356 & 418 & 0.1 & $2,5-$-IprOBDPI & 392 & 488 & 12.7 \\
\hline
\end{tabular}

Table S3. Kamlet-Taft solvent parameter set.

\begin{tabular}{|c|c|c|c|}
\hline \multirow{2}{*}{ solvent } & \multicolumn{3}{|c|}{ Kamlet-Taft solvent parameter } \\
\cline { 2 - 4 } & $\alpha$ & $\beta$ & $\pi^{*}$ \\
\hline Hex & 00 & 00 & -04 \\
\hline EA & 00 & 45 & 55 \\
\hline DOX & 00 & 37 & 55 \\
\hline ACT & 08 & 43 & 71 \\
\hline ACN & 19 & 40 & 75 \\
\hline DMF & 00 & 69 & 88 \\
\hline DMSO & 00 & 76 & 100 \\
\hline BtOH & 84 & 84 & 47 \\
\hline PrOH & 84 & 90 & 52 \\
\hline
\end{tabular}




\begin{tabular}{|c|c|c|c|}
\hline $\mathrm{EtOH}$ & 86 & 75 & 54 \\
\hline $\mathrm{MeOH}$ & 98 & 66 & 60 \\
\hline
\end{tabular}

Table S4. UV-Vis absorption $\left(\lambda_{\mathrm{ab}}\right)$ and fluorescence emission $\left(\lambda_{\mathrm{f}}\right)$ maxima (in $\mathrm{nm}$ ), extinction coefficient $(\varepsilon, \mathrm{M} \mathrm{cm})^{-1}$, fluorescence quantum yields $(\Phi, \%)$ and lifetime $(\tau, \mathrm{ns}), \mathrm{Kr}$ and $\mathrm{Knr}$ values (108 S-1) of 2,5-dialkoxy substituated chromophores in in different solvents.

\begin{tabular}{|c|c|c|c|c|c|}
\hline Sample & Subject & Hex & EA & ACN & MeOH \\
\hline 2,5-EtO & $\lambda_{\mathrm{ab}}(\varepsilon) / \lambda_{\mathrm{f}}$ & $394(2$ & $394(13$ & $396(14$ & $399(10500) / 550$ \\
BDPI & & $700) / 450$ & $500) / 483$ & $000) / 513$ & \\
\cline { 2 - 6 } & $\Phi_{\mathrm{f}} / \tau$ & $10.2 / 0.93$ & $11.1 / 1.54$ & $13.8 / 2.32$ & $2.3 / 1.10$ \\
\cline { 2 - 6 } & $\mathrm{Kr} / \mathrm{Knr}$ & $1.09 / 9.66$ & $0.72 / 5.77$ & $0.60 / 3.71$ & $0.21 / 8.89$ \\
\hline \multirow{2}{*}{$\begin{array}{c}2,5-\mathrm{Hex} \\
\text { OBDPI }\end{array}$} & $\lambda_{\mathrm{ab}}(\varepsilon) / \lambda_{\mathrm{f}}$ & $396(11$ & $396(12$ & $397(11$ & $401(10200) / 550$ \\
& & $900) / 450$ & $000) / 483$ & $200) / 513$ & \\
\cline { 2 - 6 } & $\Phi_{\mathrm{f}} / \tau$ & $10.6 / 1.07$ & $11.4 / 1.57$ & $14.2 / 2.47$ & $2.3 / 1.09$ \\
\cline { 2 - 6 } & $\mathrm{Kr} / \mathrm{Knr}$ & $0.99 / 8.36$ & $0.72 / 5.65$ & $0.57 / 3.47$ & $0.21 / 8.96$ \\
\hline 2,5-IprO & $\lambda_{\mathrm{ab}}(\varepsilon) / \lambda_{\mathrm{f}}$ & $394(1$ & $393(12$ & $394(12$ & $401(11100) / 552$ \\
$\mathrm{BDPI}$ & & $900) / 451$ & $200) / 489$ & $100) / 518$ & \\
\cline { 2 - 6 } & $\Phi_{\mathrm{f}} / \tau$ & $11.5 / 1.21$ & $12.7 / 1.61$ & $15.6 / 2.71$ & $2.6 / 1.06$ \\
\cline { 2 - 6 } & $\mathrm{Kr} / \mathrm{Knr}$ & $0.95 / 7.32$ & $0.79 / 5.42$ & $0.57 / 3.12$ & $0.25 / 9.19$ \\
\hline
\end{tabular}

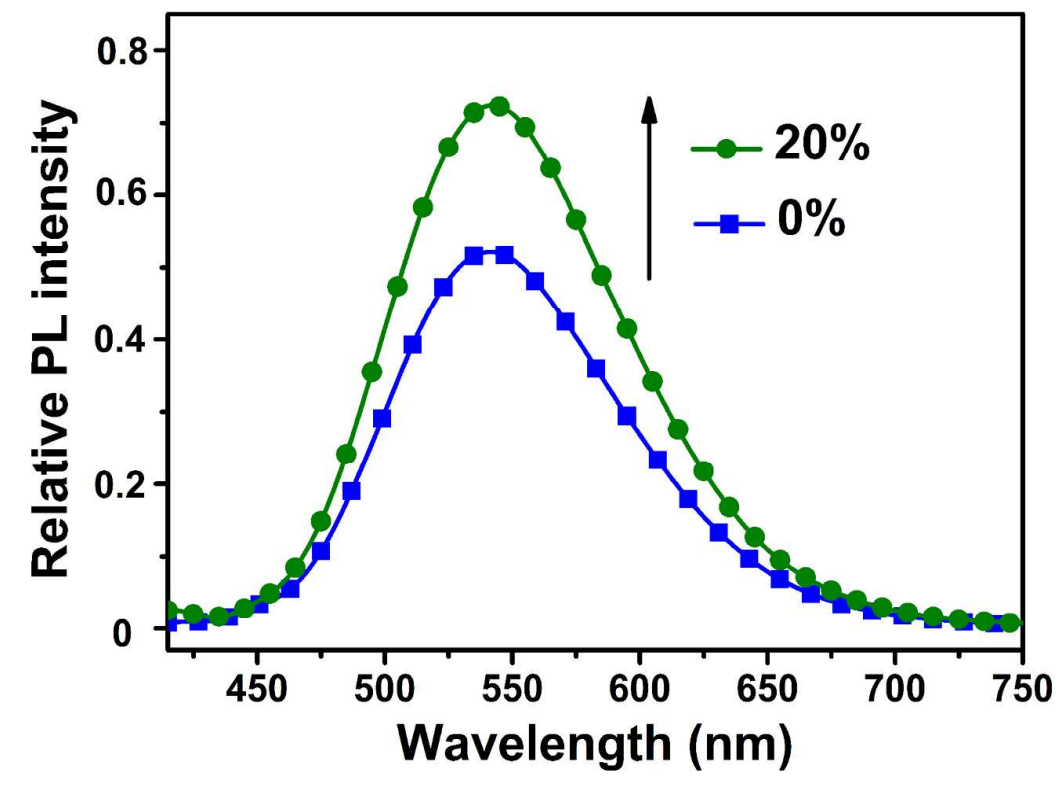

Figure S1. The fluorescence emission spectra of 2,5-MeOBDPI in $\mathrm{MeOH}$ and $\mathrm{MeOH} / \mathrm{MeOD}$, concentration $=10 \mu \mathrm{M}, \lambda_{\mathrm{ex}}=400 \mathrm{~nm}, \mathrm{MeOD}$ contents are given $(\%)$. 

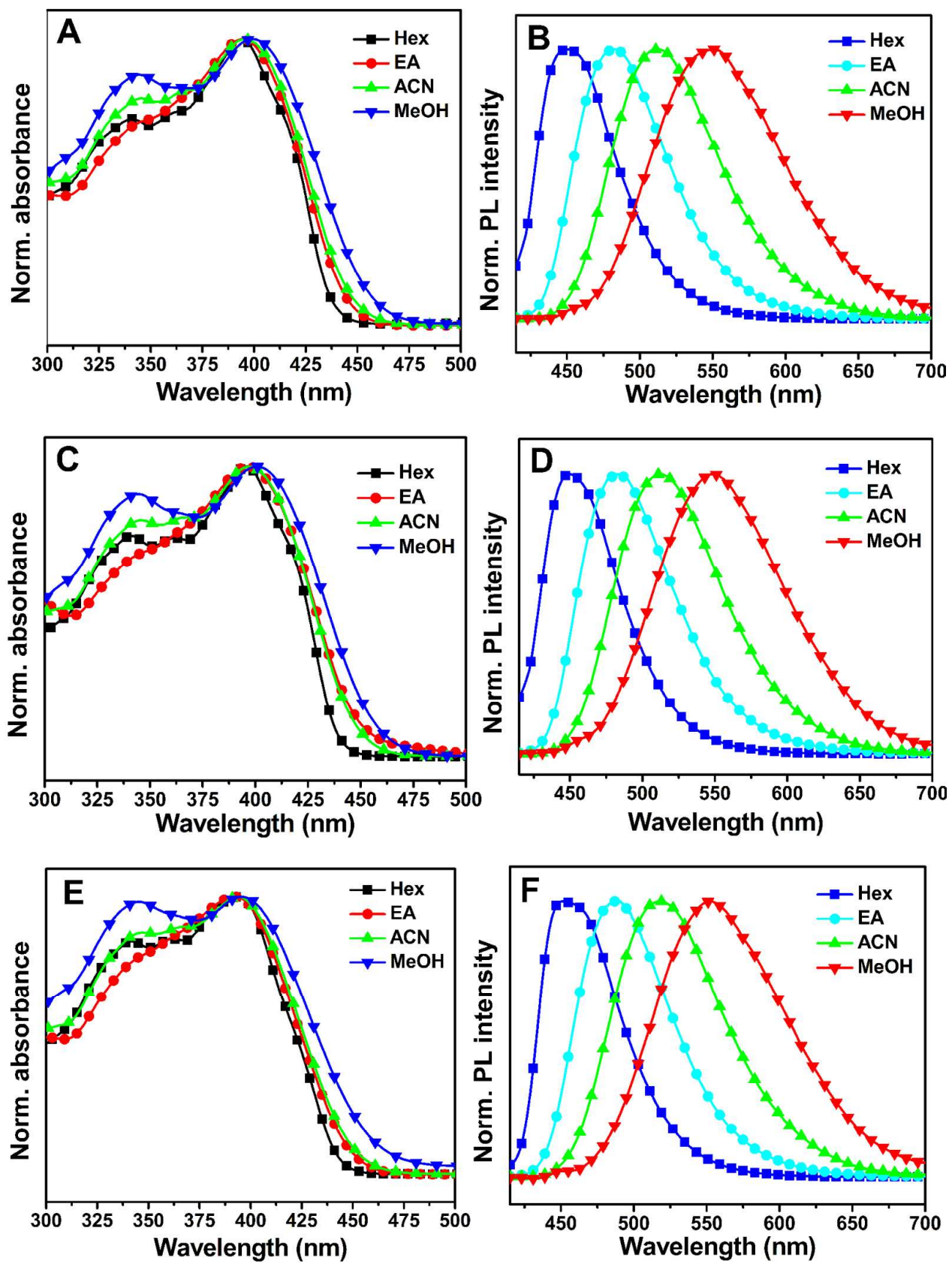

Figure S2. The normalized UV-Vis absorption (A, C, E) and fluorescence emission (B, D, F) spectra of 2,5-EtOBDPI (A, B), 2,5-HexOBDPI (C, D) and 2,5-IprOBDPI (E, F) in different solvents. 

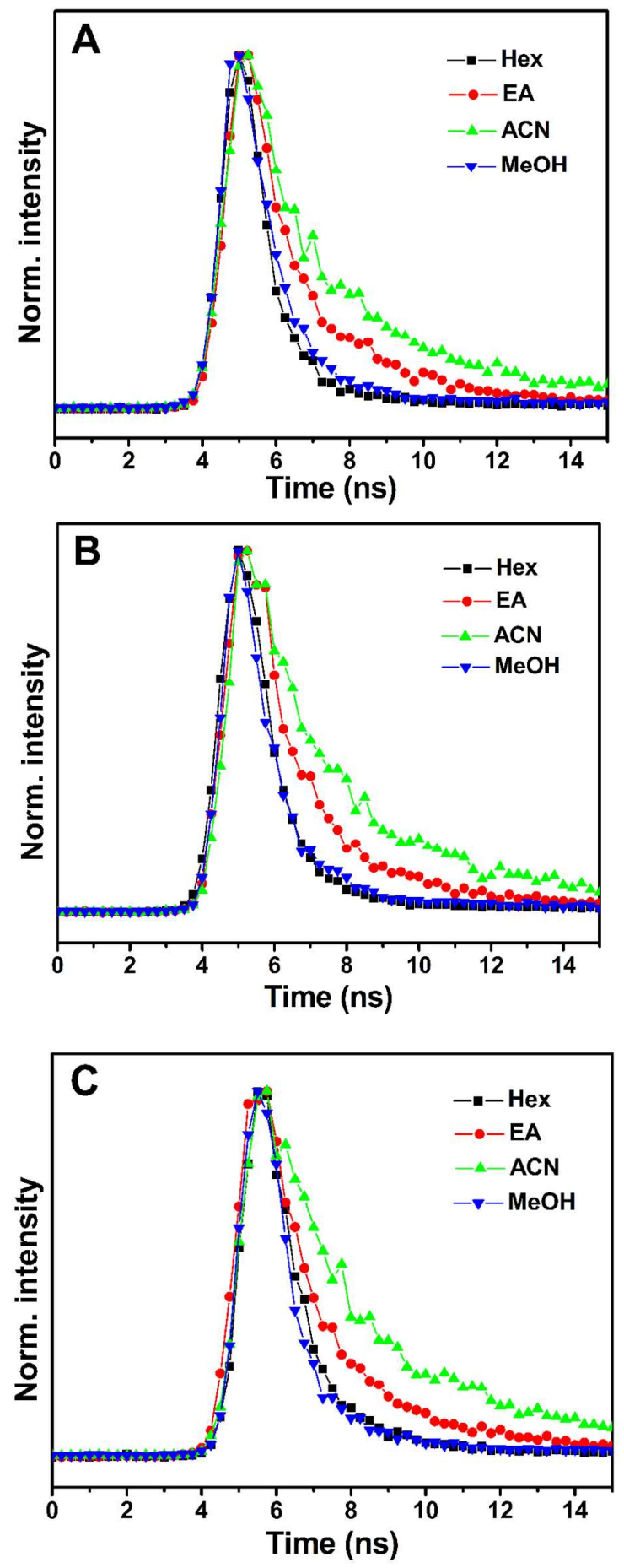

Figure S3. The time-resolved fluorescence of 2,5-EtOBDPI (A), 2,5-HexOBDPI (B) and 2,5-IprBDPI (C) in different solvents excited at $\lambda_{\max }$ of absorption and monitored at $\lambda_{\max }$ of fluorescence emission. 

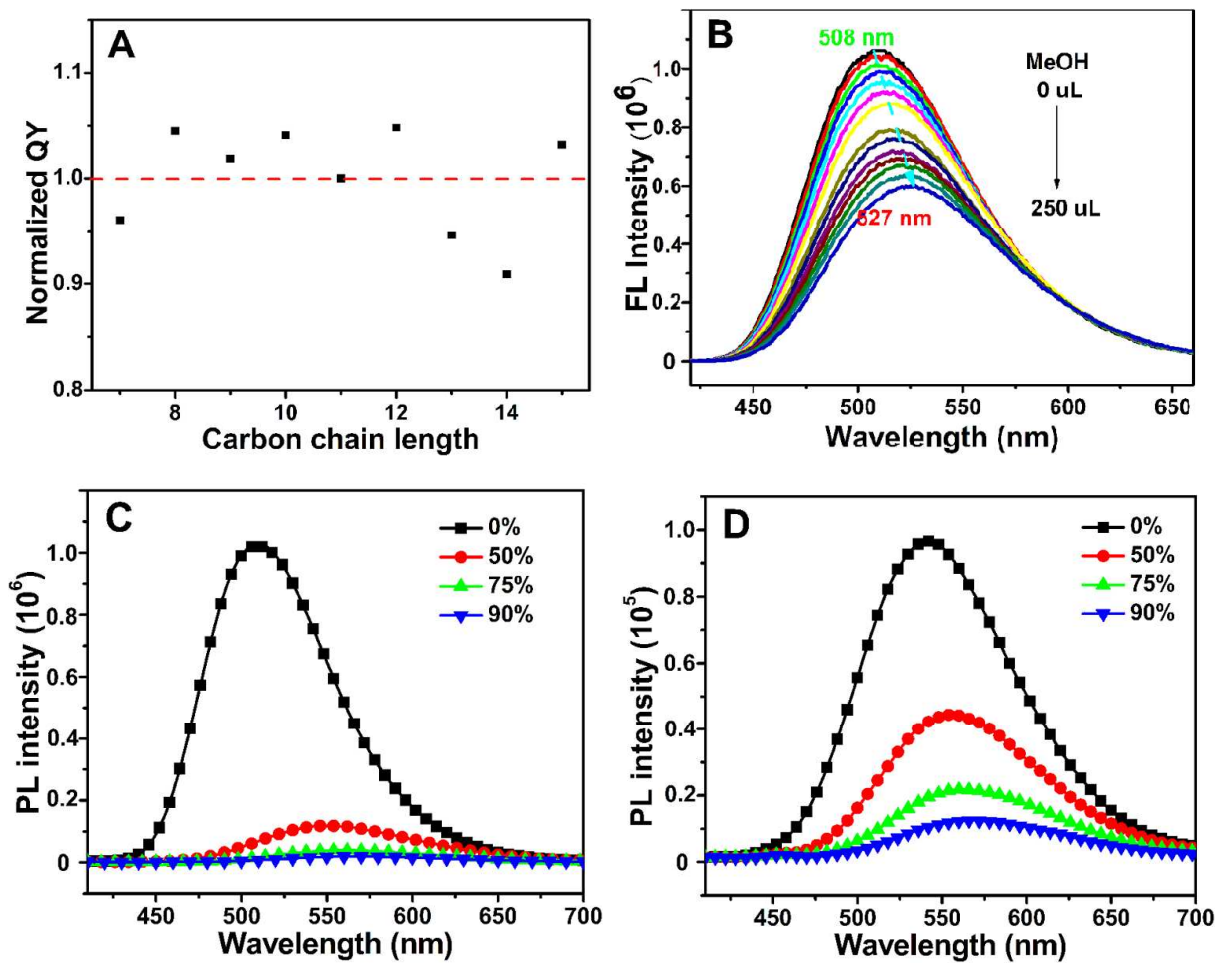

Figure S4. (A) The fluorescence QY of 2,5-MeOBDPI in various $n$-alkanes, normalized to an $n$-undecane solution. (B) The emission spectra of 2,5-MeOBDPI with $\mathrm{MeOH}$ titration from 0 to $250 \mu \mathrm{L}$ in $2.0 \mathrm{~mL} \mathrm{ACN}$; concentration $=10 \mu \mathrm{M}$. $\lambda_{\mathrm{ex}}=400 \mathrm{~nm}$; slits: $2.4 / 2.4 \mathrm{~nm}$. The emission of 2,5-MeOBDPI in DMSO/ $\mathrm{H}_{2} \mathrm{O}(\mathrm{C})$ and $\mathrm{MeOH} / \mathrm{H}_{2} \mathrm{O}$ (D) mixed solvents; water contents are given; concentration $=5 \mu \mathrm{M}$. $\lambda_{\mathrm{ex}}=400 \mathrm{~nm}$; slits: $3.2 / 3.2 \mathrm{~nm}$.
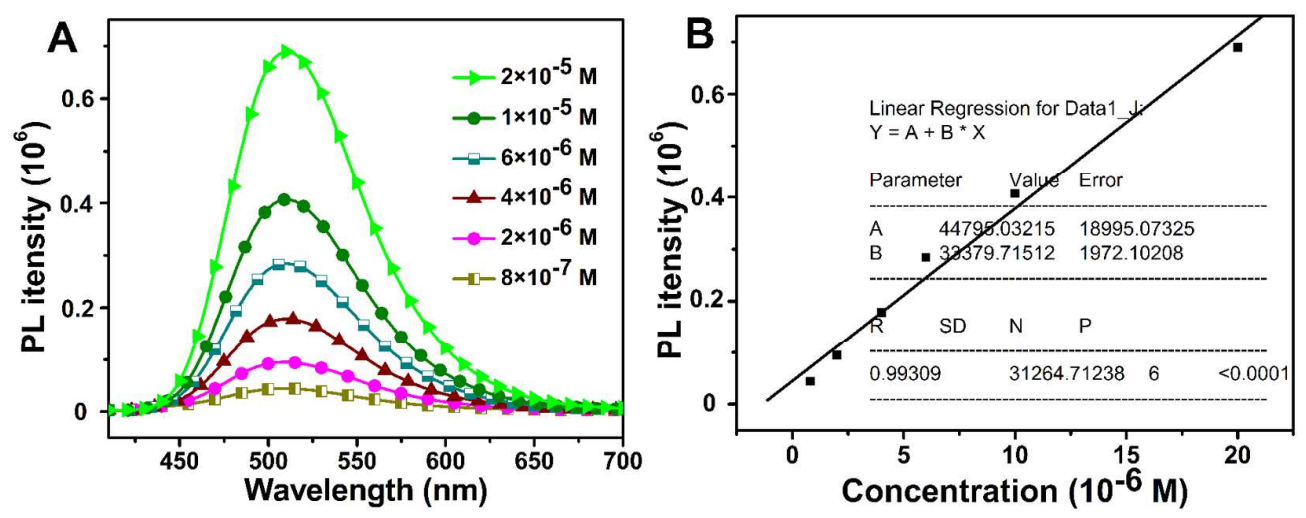

Figure S5. Linear dependence of fluorescence intensity on concentration for 2,5-MeOBDPI in DMSO. (A) The fluorescence emission spectra excited at $400 \mathrm{~nm}$, slits: 1.4/1.4 nm; (B) the corresponding linear fitting data. 


\section{Computational Details}

All of the calculations reported here were performed with the Gaussian09 program. ${ }^{[3]}$ Geometry optimization of 17 structures was carried out at B3LYP/6-31+G* without any symmetry restriction. ${ }^{[4-6]}$ After the geometry optimization was performed, analytical vibration frequencies were calculated at the same level to determine the nature of the located stationary point. We also perform a density functional theory (DFT/B3LYP/6-31+G*) to optimize the first excited state (time-dependent DFT formulation) as well as to calculate vibrational normal modes for the 17 chromophores. ${ }^{[7,8]}$ In addition, the 2,5-MeOBDPI molecule was also optimized by including the solvent effect through the polarizable continuum model (PCM). In the PCM method the solvent is treated as an infinite continuum dielectric and the solvent response to the presence of the electric field generated by the solute charge distribution (assumed to be contained in a volume of known form and dimension, the molecular cavity) is represented in terms of an apparent charge spread on the cavity surface. The solvents chosen in these calculations are methanol, cyclohexane (instead of hexane), acetonitrile and DMSO, and the solvent reaction field was equilibrated to the solute electronic density also in the calculation of excited states.
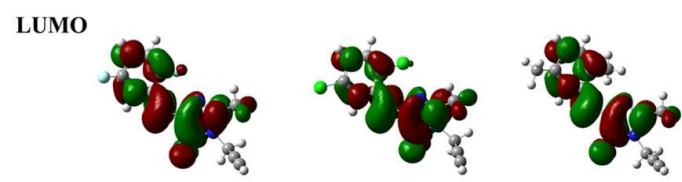

номо
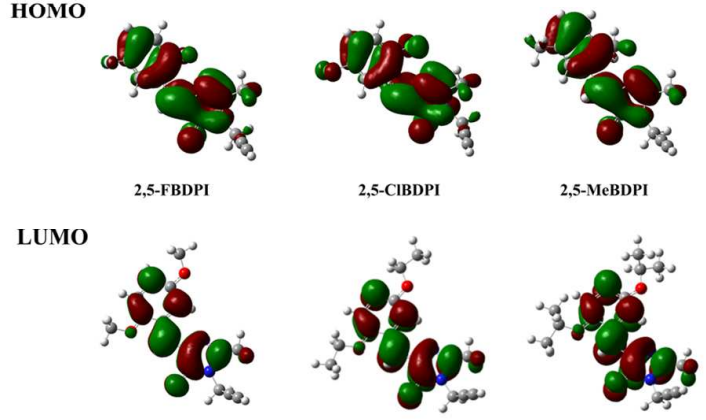

Hомо
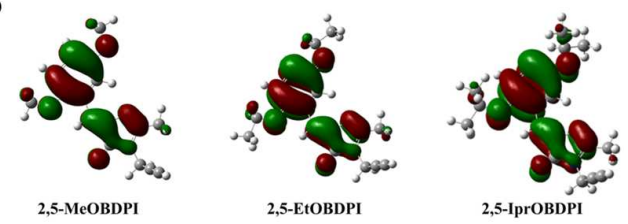

Figure S6. Molecular orbital diagrams for the (top) LUMOs and (bottom) HOMOs of 2,5-disubstituated GFP-like chromophores derivatives obtained from DFT calculations. 


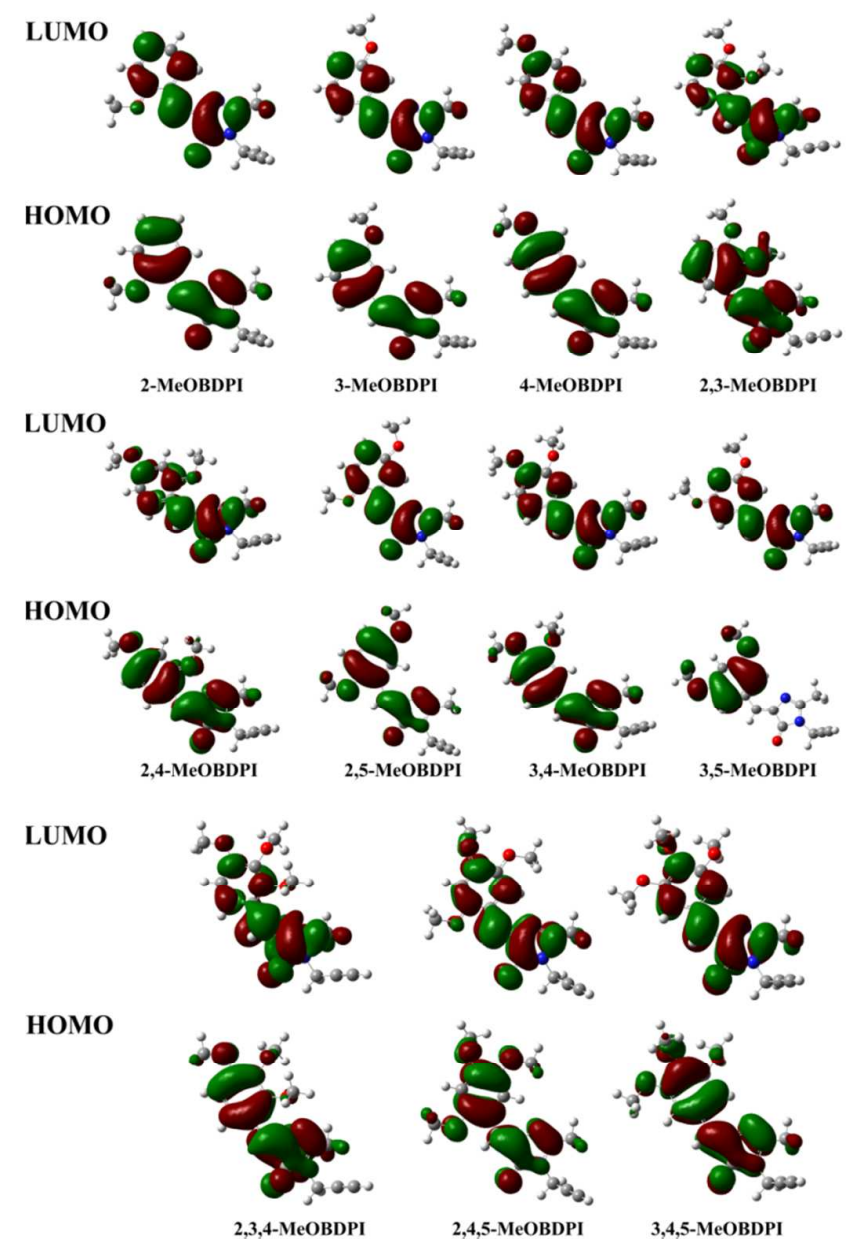

Figure S7. Molecular orbital diagrams for the (top) LUMOs and (bottom) HOMOs of methoxyl-substituted GFPc analogs obtained from DFT calculations.

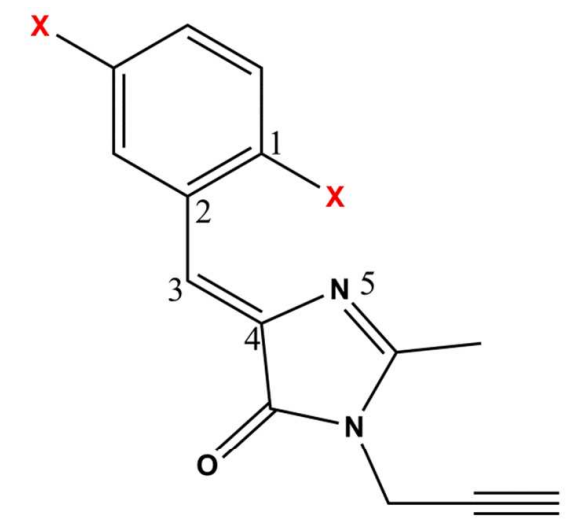

$$
\mathrm{X}=\mathrm{F}, \mathrm{Cl}, \mathrm{CH}_{3}, \mathrm{CH}_{3} \mathrm{O}, \mathrm{C}_{2} \mathrm{H}_{5} \mathrm{O}, \mathrm{C}_{3} \mathrm{H}_{7} \mathrm{O}, \mathrm{C}_{6} \mathrm{H}_{13} \mathrm{O}
$$

Figure S8. The scheme to illustrate the rotational angle between the aromatic ring and the imidazolinone heterocyclic ring. 
Table S5. The torsional angle between the aromatic ring and the imidazolinone heterocyclic ring at 2,5-disubstituted position and dipole moment of $\mathrm{S}_{0}$ and $\mathrm{S}_{1}$ states.

\begin{tabular}{|c|c|c|c|c|c|c|c|c|}
\hline \multicolumn{2}{|c|}{ Substituents } & $\mathrm{F}-$ & $\mathrm{Cl}-$ & $\mathrm{CH}_{3-}$ & $\mathrm{CH}_{3} \mathrm{O}-$ & $\mathrm{C}_{2} \mathrm{H}_{5} \mathrm{O}-$ & $\mathrm{C}_{3} \mathrm{H}_{7}-$ & $\mathrm{C}_{6} \mathrm{H}_{13} \mathrm{O}-$ \\
\hline \multirow{3}{*}{$\mathrm{S}_{0}$} & $\tau_{1-2-3-4}$ & 39.68 & 53.44 & 42.23 & 179.78 & 179.53 & 179.69 & 179.85 \\
\cline { 2 - 9 } & $\tau_{2-3-4-5}$ & 2.74 & 1.04 & 2.01 & 0.17 & 0.12 & 0.20 & 0.17 \\
\cline { 2 - 10 } & Dipole & 3.52 & 3.64 & 2.81 & 2.80 & 2.77 & 2.87 & 2.79 \\
\hline \multirow{3}{*}{$\mathrm{S}_{1}$} & $\tau_{1-2-3-4}$ & 8.89 & 13.60 & 63.07 & 81.50 & 81.39 & 81.59 & 82.41 \\
\cline { 2 - 10 } & $\tau_{2-3-4-5}$ & 18.58 & 0.56 & 16.24 & 6.94 & 5.57 & 5.66 & 6.75 \\
\cline { 2 - 10 } & Dipole & 6.72 & 3.67 & 9.17 & 16.81 & 16.70 & 16.94 & 4.47 \\
\hline
\end{tabular}

Table S6. The Mulliken atomic charge and charge transfer $\left(\Delta=\mathrm{S}_{1}-\mathrm{S}_{0}\right)$ upon excitation $\left(\mathrm{S}_{0}-\mathrm{S}_{1}\right)$ for the aromatic ring and the imidazolinone heterocyclic ring of 2,5-MeOBDPI.

\begin{tabular}{|c|c|c|c|c|c|c|c|c|}
\hline \multicolumn{2}{|c|}{ Aromatic ring (a) } & 2,5-FB & 2,5-ClB & 2,5-MeB & 2,5-MeO & 2,5-EtO & 2,5-Hex & 2,5-IprO \\
\hline \multirow{3}{*}{ charge } & $\mathrm{S}_{0}$ & 0.3683 & 1.1219 & 1.3451 & -0.2401 & -0.0881 & 0.2961 & -0.0671 \\
\hline & $\mathrm{S}_{1}$ & -0.3417 & 0.6904 & 0.9794 & 0.0506 & 0.1000 & 0.0332 & 0.1562 \\
\hline & $\Delta$ & -0.7100 & -0.4315 & -0.3657 & 0.2907 & 0.1881 & -0.2629 & 0.2233 \\
\hline \multicolumn{2}{|c|}{ Heterocycle (b) } & $\begin{array}{c}2,5-\mathrm{FB} \\
\mathrm{DPI}\end{array}$ & 2,5-ClB & 2,5-MeB & $\begin{array}{c}2,5-\mathrm{MeO} \\
\mathrm{RDPI}\end{array}$ & 2,5-EtO & 2,5-Hex & 2,5-IprO \\
\hline \multirow{3}{*}{ charge } & $\mathrm{S}_{0}$ & -0.1853 & -0.4637 & -0.9137 & -0.0172 & -0.1146 & -0.3318 & -0.1084 \\
\hline & $\mathrm{S}_{1}$ & 0.1865 & -0.2220 & -0.6096 & -0.2972 & -0.3006 & -0.0360 & -0.3080 \\
\hline & $\Delta$ & 0.3718 & 0.2417 & 0.3041 & -0.2800 & -0.1860 & 0.2958 & -0.1996 \\
\hline
\end{tabular}

Table S7. The dipole moment of $\mathrm{S}_{0}$ and $\mathrm{S}_{1}$ states of 2,5-dialkoy substituted chromophores with 
different carbochain length $(n=1$ to 6$)$.

\begin{tabular}{|c|c|c|c|c|c|c|}
\hline $\begin{array}{c}\text { 2,5-disubstit } \\
\text { utes }\end{array}$ & $\mathrm{CH}_{3} \mathrm{O}$ & $\mathrm{CH}_{3} \mathrm{CH}_{2} \mathrm{O}$ & $\begin{array}{c}\mathrm{CH}_{3}\left(\mathrm{CH}_{2}\right)_{2} \\
\mathrm{O}\end{array}$ & $\begin{array}{c}\mathrm{CH}_{3}\left(\mathrm{CH}_{2}\right)_{3} \\
\mathrm{O}\end{array}$ & $\begin{array}{c}\mathrm{CH}_{3}\left(\mathrm{CH}_{2}\right)_{4} \\
\mathrm{O}\end{array}$ & $\begin{array}{c}\mathrm{CH}_{3}\left(\mathrm{CH}_{2}\right)_{5} \\
\mathrm{O}\end{array}$ \\
\hline $\mathrm{S}_{0}$ & 2.80 & 2.77 & 2.73 & 2.79 & 2.70 & 2.79 \\
\hline $\mathrm{S}_{1}$ & 16.81 & 16.70 & 10.66 & 10.76 & 10.67 & 4.47 \\
\hline
\end{tabular}

Table S8. The Mulliken atomic charge and charge transfer $\left(\Delta=\mathrm{S}_{1}-\mathrm{S}_{0}\right)$ upon excitation $\left(\mathrm{S}_{0}-\mathrm{S}_{1}\right)$ for the aromatic ring and the imidazolinone heterocyclic ring of 2,5-alkoy disubstitued chromophores with different carbochain length ( $n=1$ to 6$)$.

\begin{tabular}{|c|c|c|c|c|c|c|c|}
\hline \multicolumn{2}{|c|}{$\begin{array}{l}\text { Aromatic ring } \\
\text { (a) }\end{array}$} & $\mathrm{CH}_{3} \mathrm{O}$ & $\begin{array}{c}\mathrm{CH}_{3} \mathrm{CH}_{2} \\
\mathrm{O}\end{array}$ & $\begin{array}{c}\mathrm{CH}_{3}\left(\mathrm{CH}_{2}\right)_{2} \\
\mathrm{O}\end{array}$ & $\begin{array}{c}\mathrm{CH}_{3}\left(\mathrm{CH}_{2}\right)_{3} \\
\mathrm{O}\end{array}$ & $\begin{array}{c}\mathrm{CH}_{3}\left(\mathrm{CH}_{2}\right)_{4} \\
\mathrm{O}\end{array}$ & $\mathrm{CH}_{3}\left(\mathrm{CH}_{2}\right)_{5} \mathrm{O}$ \\
\hline \multirow{3}{*}{ charge } & $\mathrm{S}_{0}$ & -0.2401 & -0.0881 & 0.2769 & 0.2813 & 0.2840 & 0.2961 \\
\hline & $\mathrm{S}_{1}$ & 0.0506 & 0.1000 & 0.2472 & 0.2248 & 0.1302 & 0.0332 \\
\hline & $\Delta$ & 0.2907 & 0.1881 & -0.0297 & -0.0565 & -0.1538 & -0.2629 \\
\hline \multicolumn{2}{|c|}{ Heterocycle (b) } & $\mathrm{CH}_{3} \mathrm{O}$ & $\begin{array}{c}\mathrm{CH}_{3} \mathrm{CH}_{2} \\
\mathrm{O}\end{array}$ & $\begin{array}{c}\mathrm{CH}_{3}\left(\mathrm{CH}_{2}\right)_{2} \\
\mathrm{O}\end{array}$ & $\begin{array}{c}\mathrm{CH}_{3}\left(\mathrm{CH}_{2}\right)_{3} \\
\mathrm{O}\end{array}$ & $\begin{array}{c}\mathrm{CH}_{3}\left(\mathrm{CH}_{2}\right)_{4} \\
\mathrm{O}\end{array}$ & $\mathrm{CH}_{3}\left(\mathrm{CH}_{2}\right)_{5} \mathrm{O}$ \\
\hline \multirow{3}{*}{ charge } & $\mathrm{S}_{0}$ & -0.0172 & -0.1146 & -0.2989 & -0.3280 & -0.3480 & -0.3318 \\
\hline & $\mathrm{S}_{1}$ & -0.2972 & -0.3006 & -0.3375 & -0.3290 & -0.2590 & -0.0360 \\
\hline & $\Delta$ & -0.2800 & -0.1860 & -0.0386 & -0.0010 & 0.0890 & 0.2958 \\
\hline
\end{tabular}

Table S9. The torsional angle between the aromatic ring and the imidazolinone heterocyclic ring 
of 2,5-MeOBDPI and dipole moment of $\mathrm{S}_{0}$ and $\mathrm{S}_{1}$ states under different solvent environments.

\begin{tabular}{|c|c|c|c|c|c|}
\hline \multicolumn{2}{|c|}{ Solvent } & Methanol & Cyclohexane & Acetonitrile & DMSO \\
\hline \multirow{3}{*}{$S_{0}$} & $\tau_{1-2-3-4}$ & 179.14 & 179.95 & 179.13 & 179.11 \\
\cline { 2 - 6 } & $\tau_{2-3-4-5}$ & 0.41 & 0.05 & 0.41 & 0.42 \\
\cline { 2 - 6 } & Dipole & 3.93 & 3.21 & 3.93 & 83.92 \\
\hline \multirow{3}{*}{$S_{1}$} & $\tau_{1-2-3-4}$ & 84.00 & 82.61 & 83.97 & 1.49 \\
\cline { 2 - 6 } & $\tau_{2-3-4-5}$ & 1.44 & 4.76 & 1.45 & 21.45 \\
\cline { 2 - 6 } & Dipole & 6.04 & 18.67 & 21.40 & \multirow{2}{*}{. } \\
\hline
\end{tabular}

Table S10. The Mulliken atomic charge and charge transfer $(\Delta)$ upon excitation $\left(\mathrm{S}_{0}-\mathrm{S}_{1}\right)$ for the aromatic ring and the imidazolinone heterocyclic ring of 2,5-MeOBDPI in different solvents.

\begin{tabular}{|c|c|c|c|c|c|}
\hline \multicolumn{2}{|c|}{ Aromatic ring (a) } & Cyclohexane & Acetonitrile & DMSO & Methanol \\
\hline \multirow{2}{*}{ charge } & $\mathrm{S}_{0}$ & -0.2285 & -0.2017 & -0.2011 & -0.2020 \\
\cline { 2 - 6 } & $\mathrm{S}_{1}$ & 0.1621 & 0.3621 & 0.3666 & -0.3951 \\
\cline { 2 - 6 } & $\Delta$ & 0.3906 & 0.5638 & 0.5677 & -0.1931 \\
\hline \multirow{2}{*}{ Heterocycle (b) } & Cyclohexane & Acetonitrile & DMSO & Methanol \\
\hline \multirow{2}{*}{ charge } & $\mathrm{S}_{0}$ & -0.0088 & 0.0122 & 0.0128 & 0.0120 \\
\cline { 2 - 6 } & $\mathrm{S}_{1}$ & -0.3912 & -0.5737 & -0.5773 & -0.1306 \\
\cline { 2 - 6 } & $\Delta$ & -0.3824 & -0.5859 & -0.5901 & -0.1426 \\
\hline
\end{tabular}

\section{4. ${ }^{1} \mathrm{H}$ and ${ }^{13} \mathrm{C}$ NMR Spectra for GFPc Analogs}




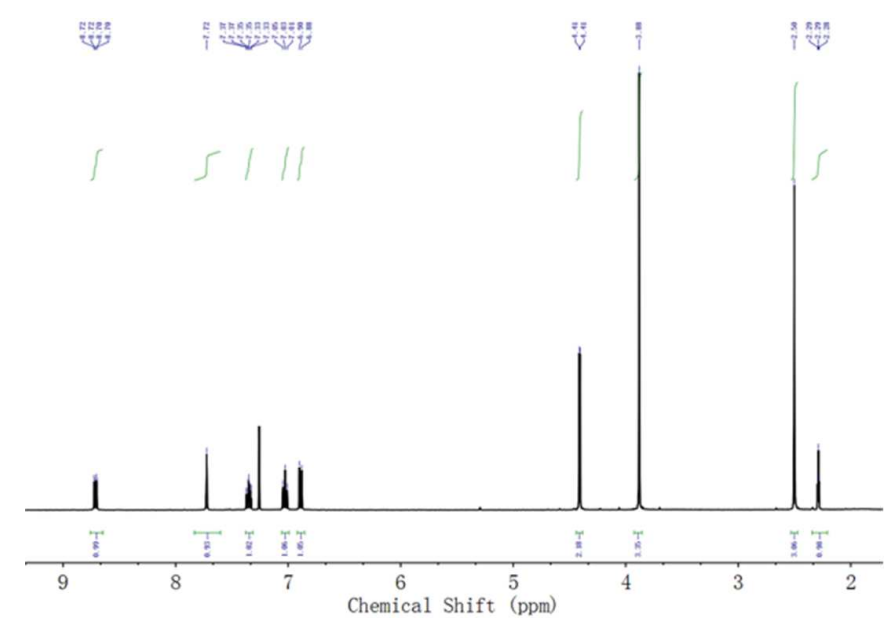

Figure S9. The ${ }^{1} \mathrm{H}$ NMR spectrum of 2-MeOBDPI.
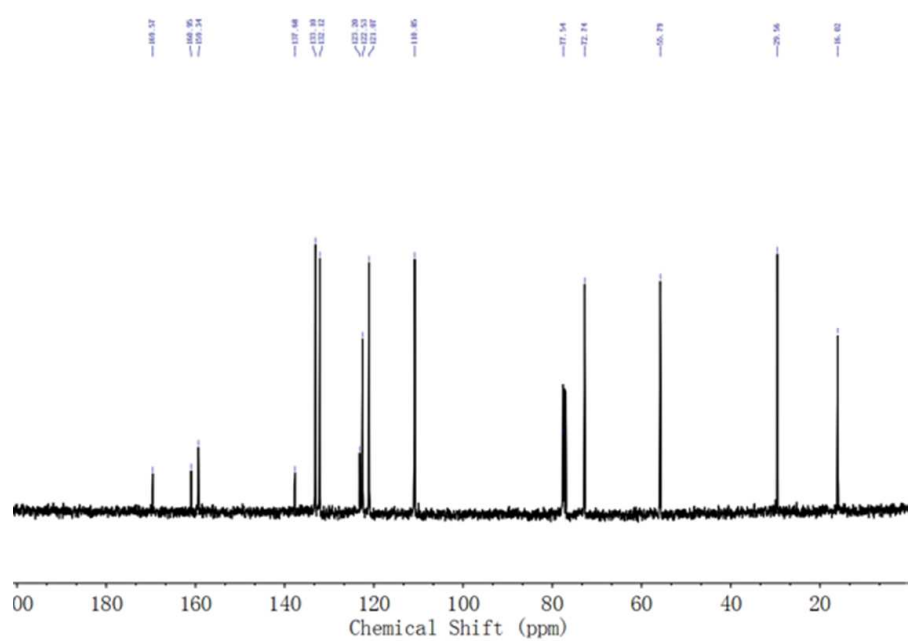

Figure S10. The ${ }^{13} \mathrm{C}$ NMR spectrum of 2-MeOBDPI.

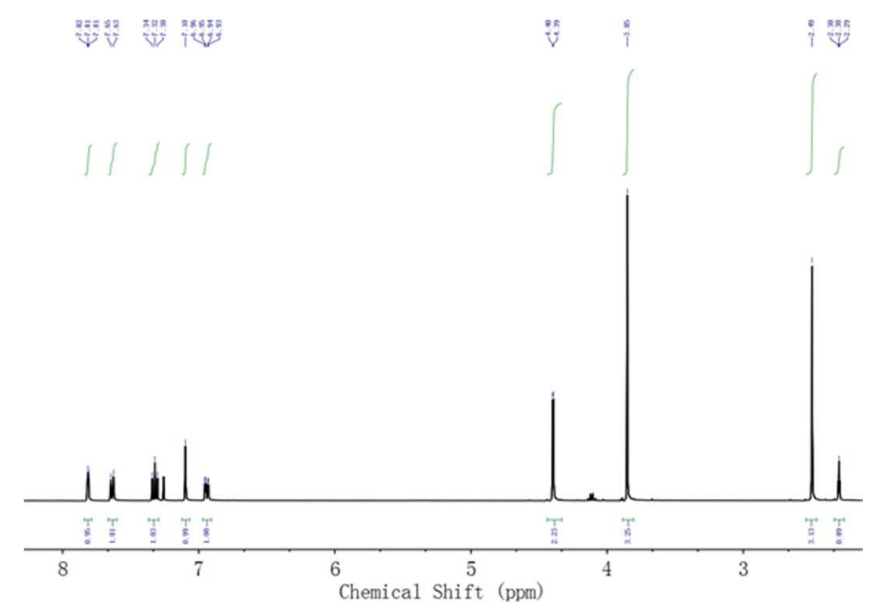

Figure S11. The ${ }^{1} \mathrm{H}$ NMR spectrum of 3-MeOBDPI. 

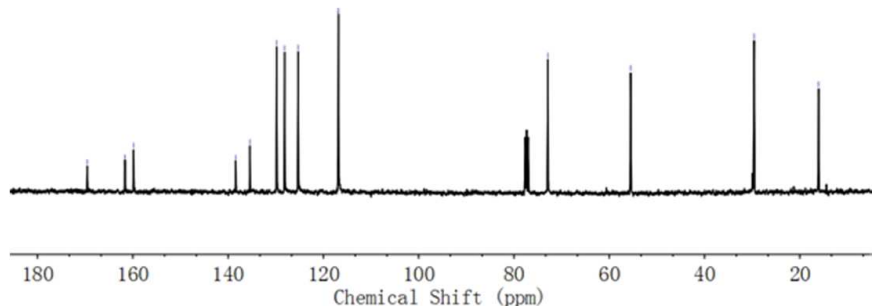

Figure S12. The ${ }^{13} \mathrm{C}$ NMR spectrum of 3-MeOBDPI.
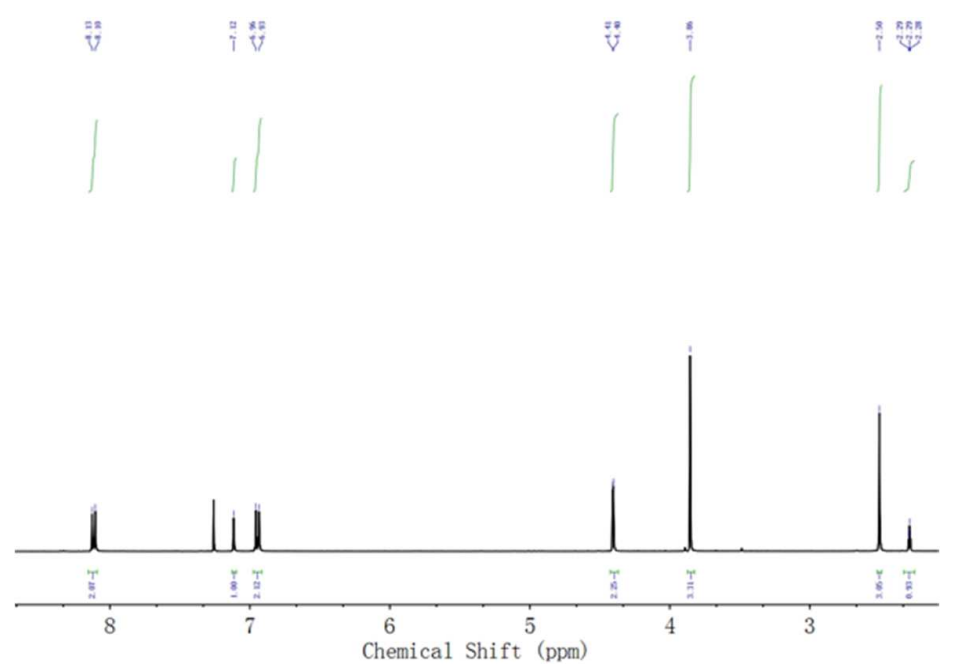

Figure S13. The ${ }^{1} \mathrm{H}$ NMR spectrum of 4-MeOBDPI.
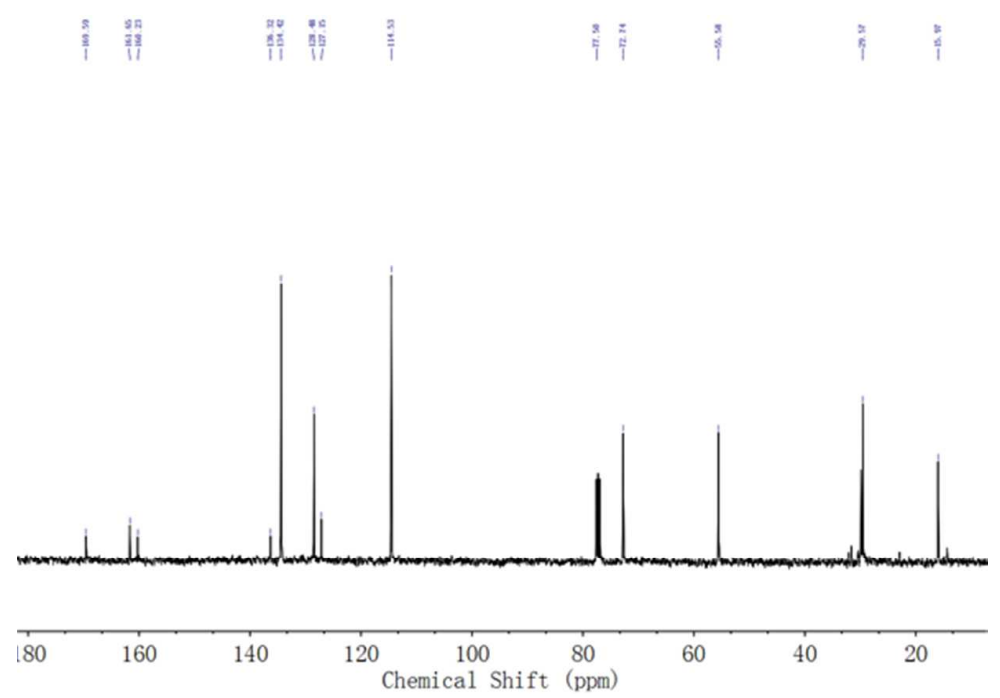

Figure S14. The ${ }^{13} \mathrm{C}$ NMR spectrum of 4-MeOBDPI. 


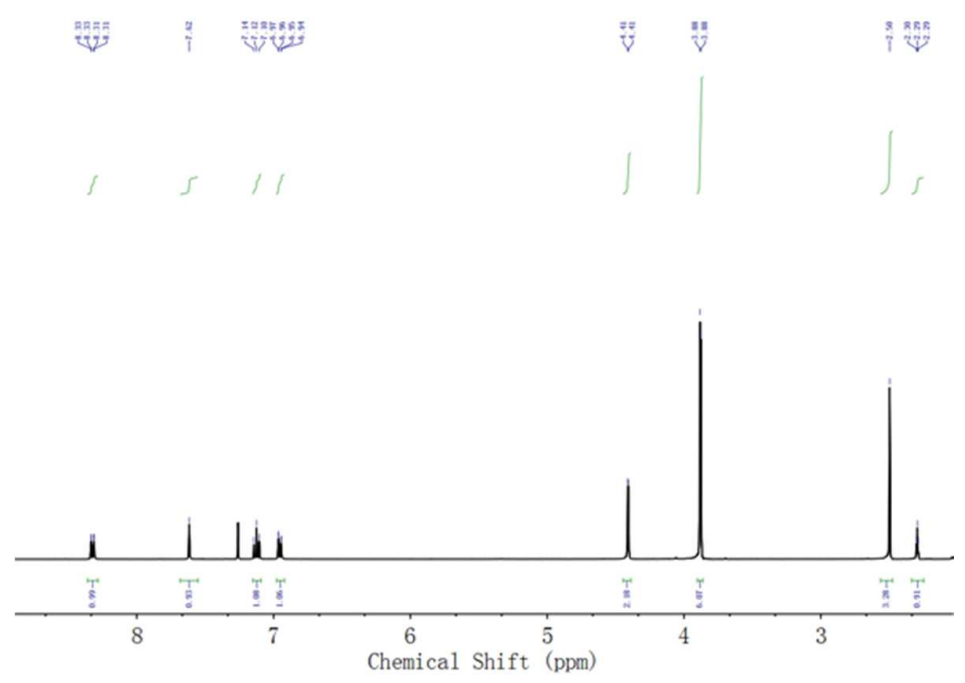

Figure S15. The ${ }^{1} \mathrm{H}$ NMR spectrum of 2,3-MeOBDPI.
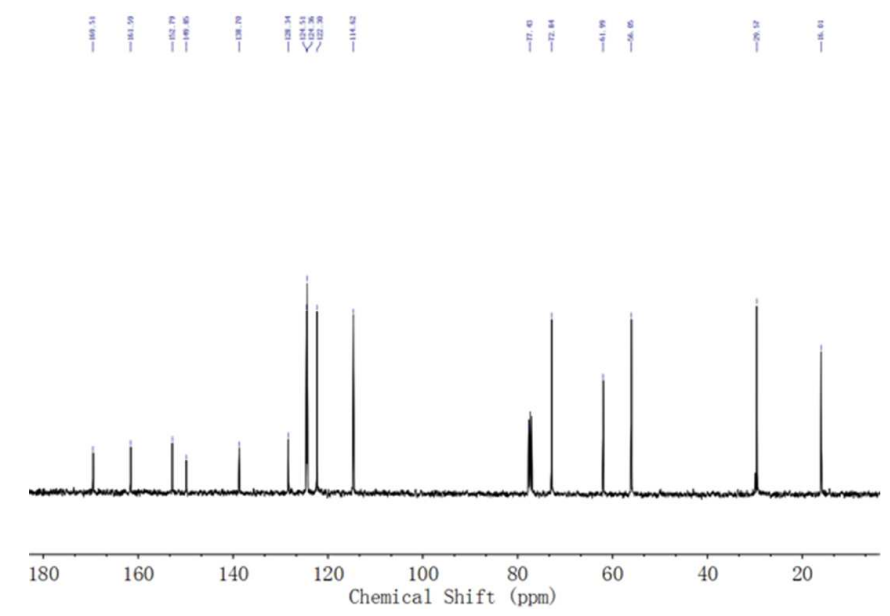

Figure S16. The ${ }^{13} \mathrm{C}$ NMR spectrum of 2,3-MeOBDPI.

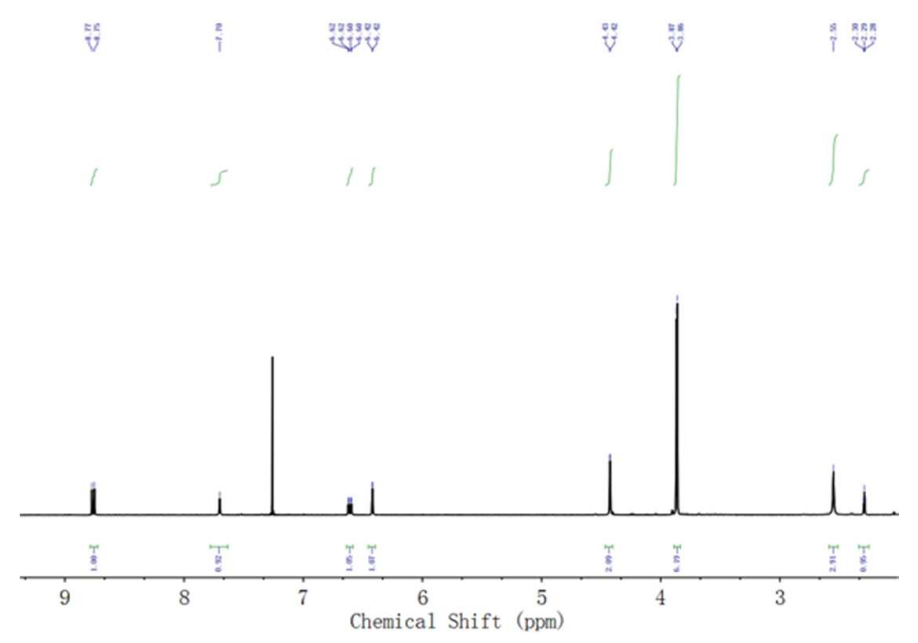

Figure S17. The ${ }^{1} \mathrm{H}$ NMR spectrum of 2,4-MeOBDPI. 

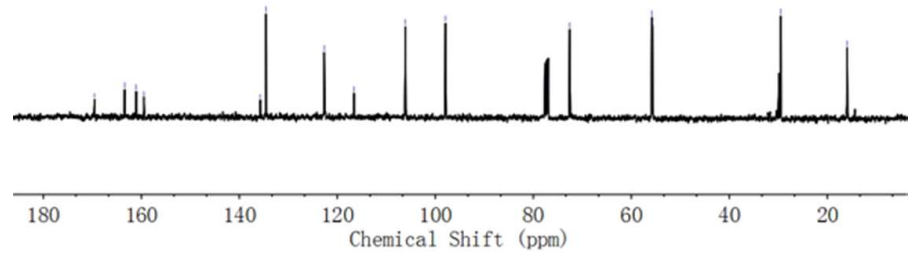

Figure S18. The ${ }^{13} \mathrm{C}$ NMR spectrum of 2,4-MeOBDPI.
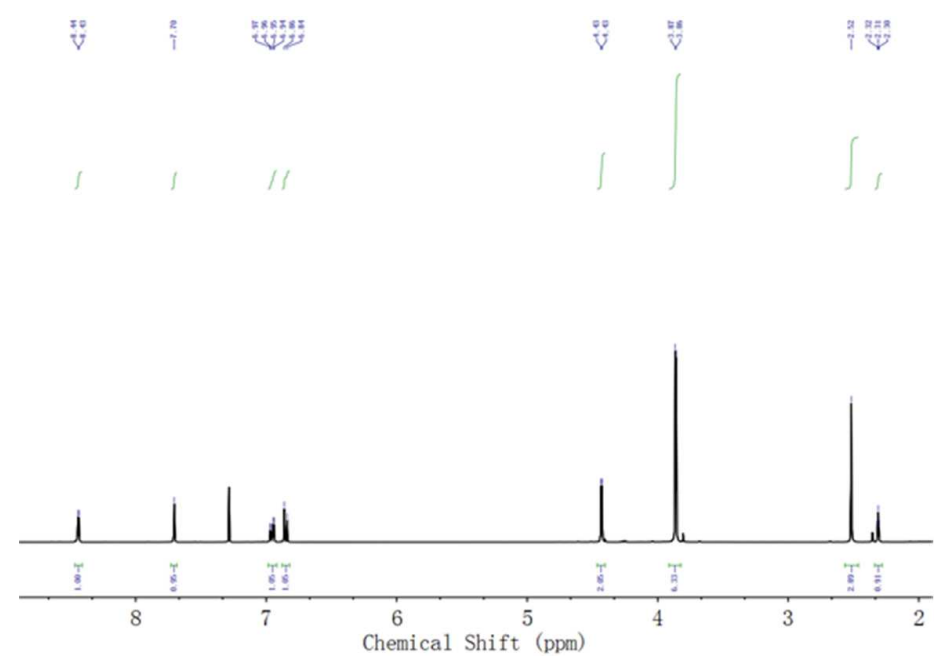

Figure S19. The ${ }^{1} \mathrm{H}$ NMR spectrum of 2,5-MeOBDPI.
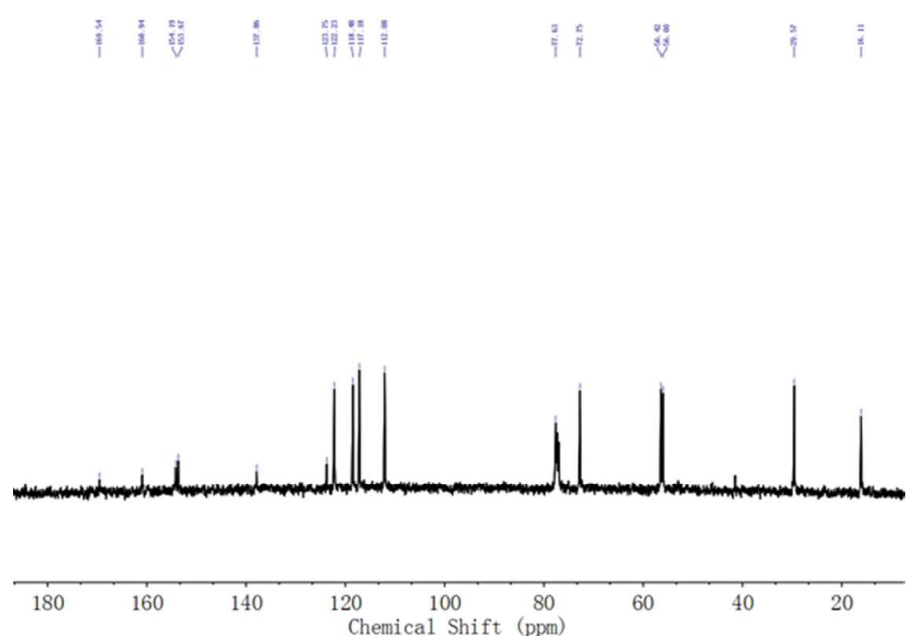

Figure S20. The ${ }^{13} \mathrm{C}$ NMR spectrum of 2,5-MeOBDPI. 


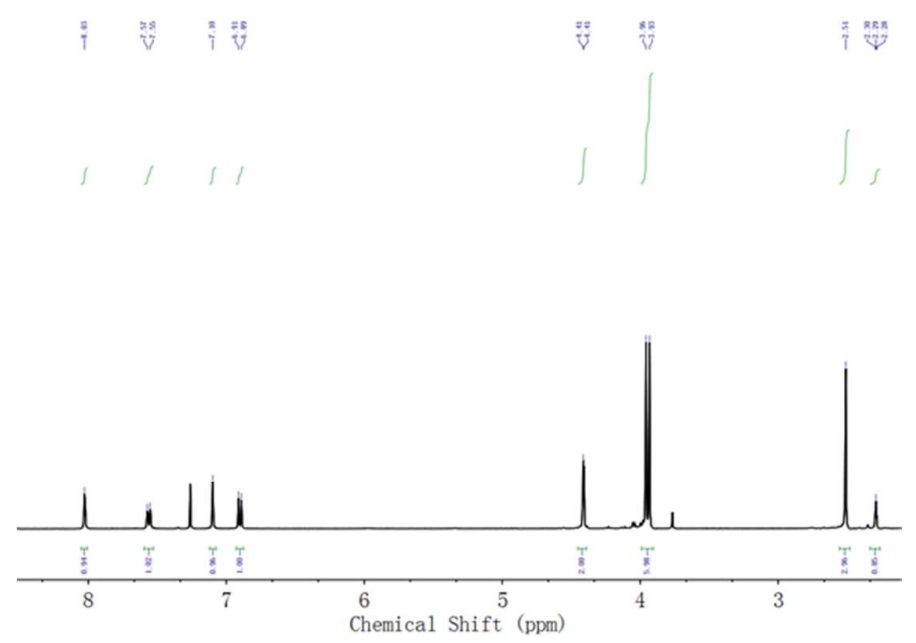

Figure S21. The ${ }^{1} \mathrm{H}$ NMR spectrum of 3,4-MeOBDPI.
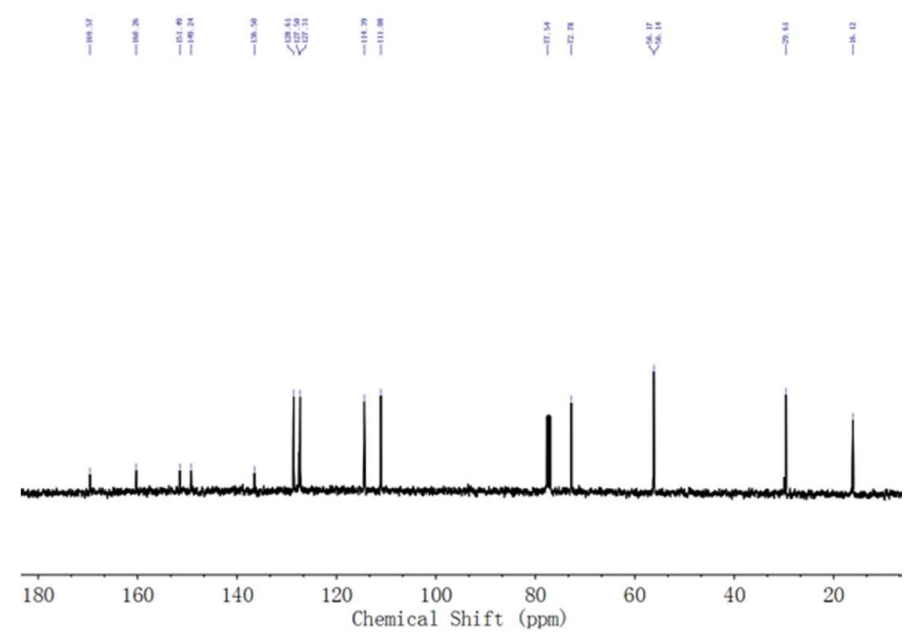

Figure S22. The ${ }^{13} \mathrm{C}$ NMR spectrum of 3,4-MeOBDPI.

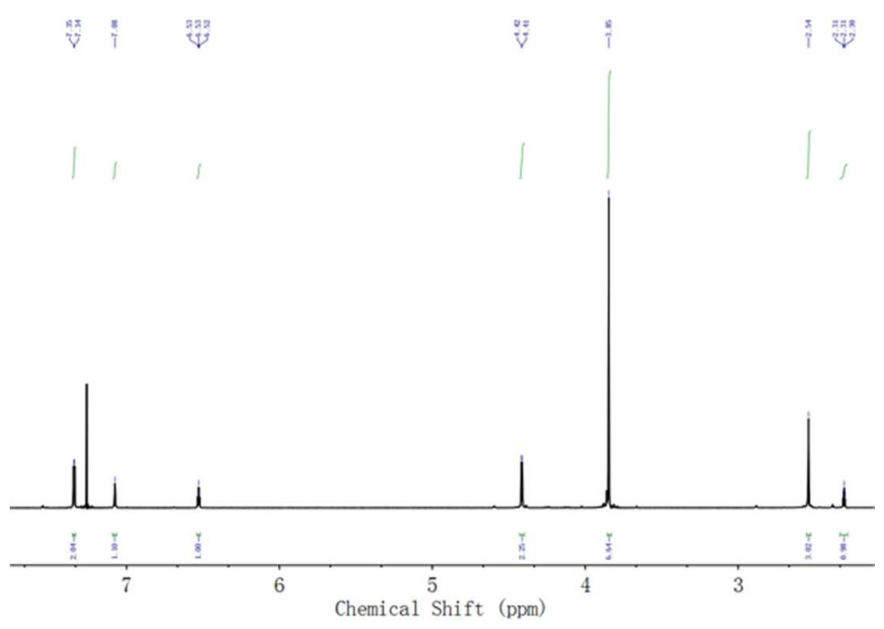

Figure S23. The ${ }^{1} \mathrm{H}$ NMR spectrum of 3,5-MeOBDPI. 


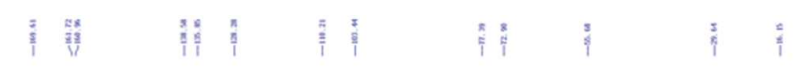

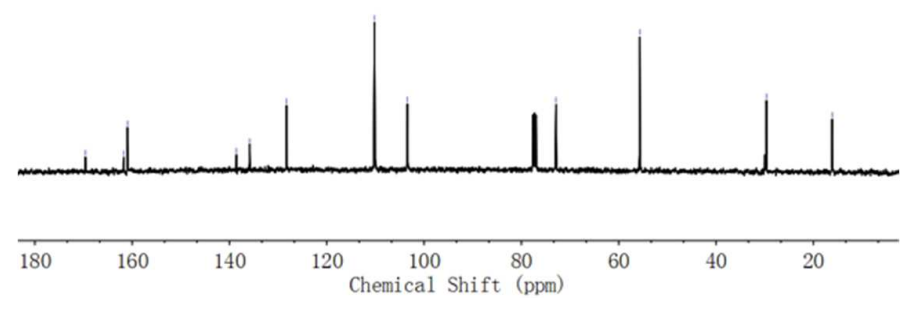

Figure S24. The ${ }^{13} \mathrm{C}$ NMR spectrum of 3,5-MeOBDPI.
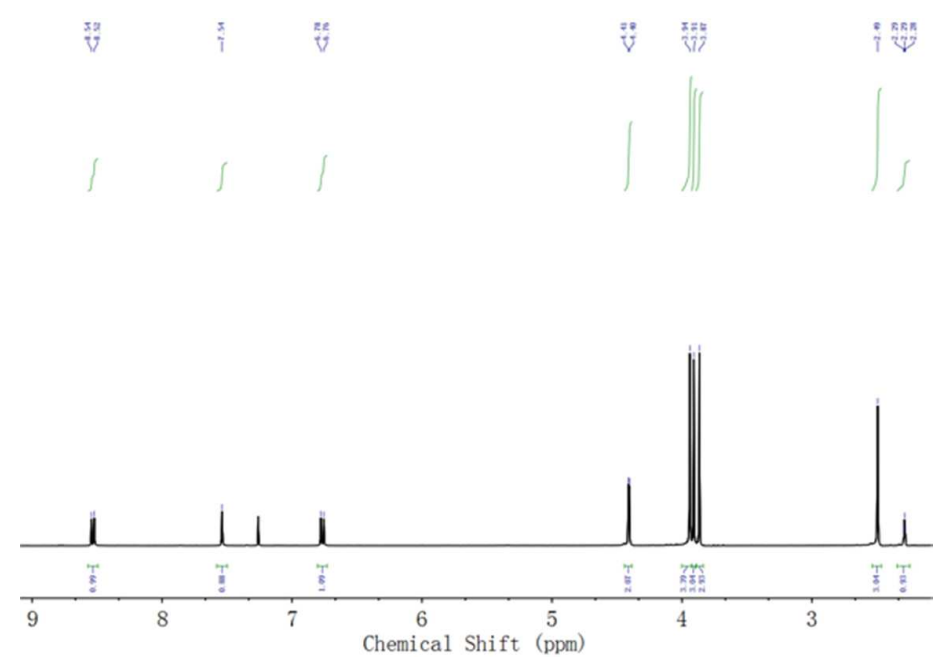

Figure S25. The ${ }^{1} \mathrm{H}$ NMR spectrum of 2,3,4-MeOBDPI.
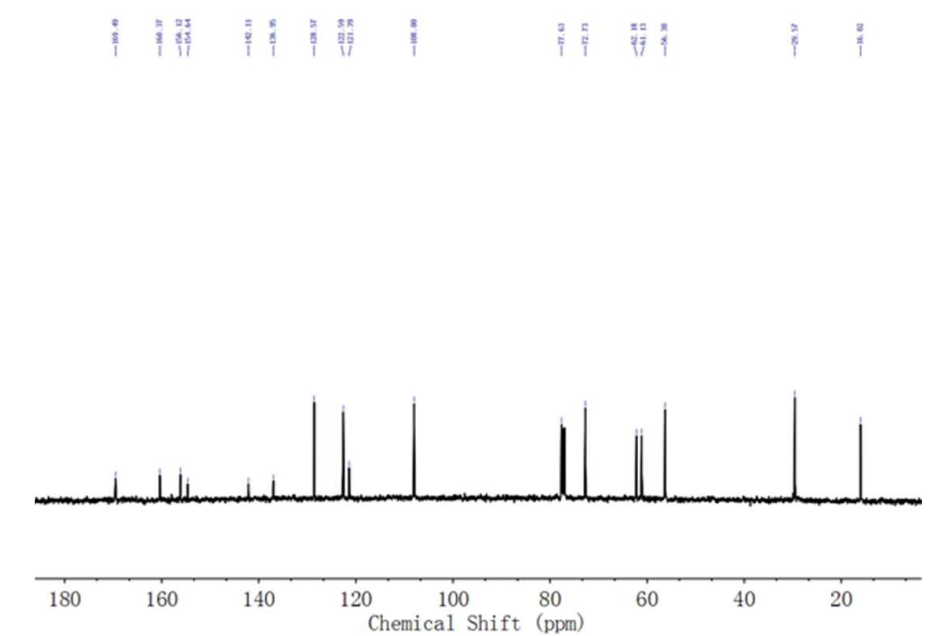

Figure S26. The ${ }^{13} \mathrm{C}$ NMR spectrum of 2,3,4-MeOBDPI. 


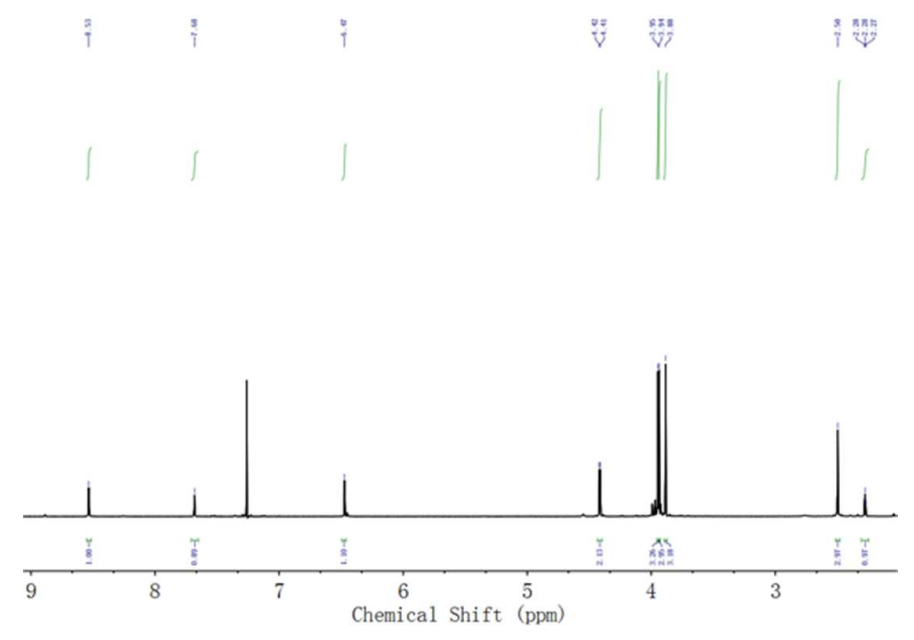

Figure S27. The ${ }^{1} \mathrm{H}$ NMR spectrum of 2,4,5-MeOBDPI.
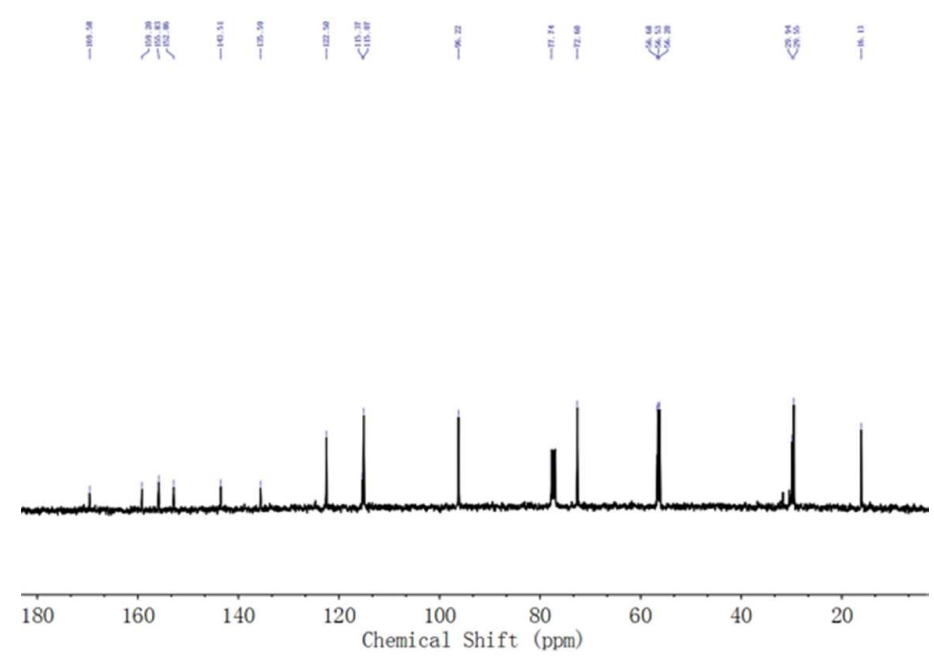

Figure S28. The ${ }^{13} \mathrm{C}$ NMR spectrum of $2,4,5-\mathrm{MeOBDPI}$.

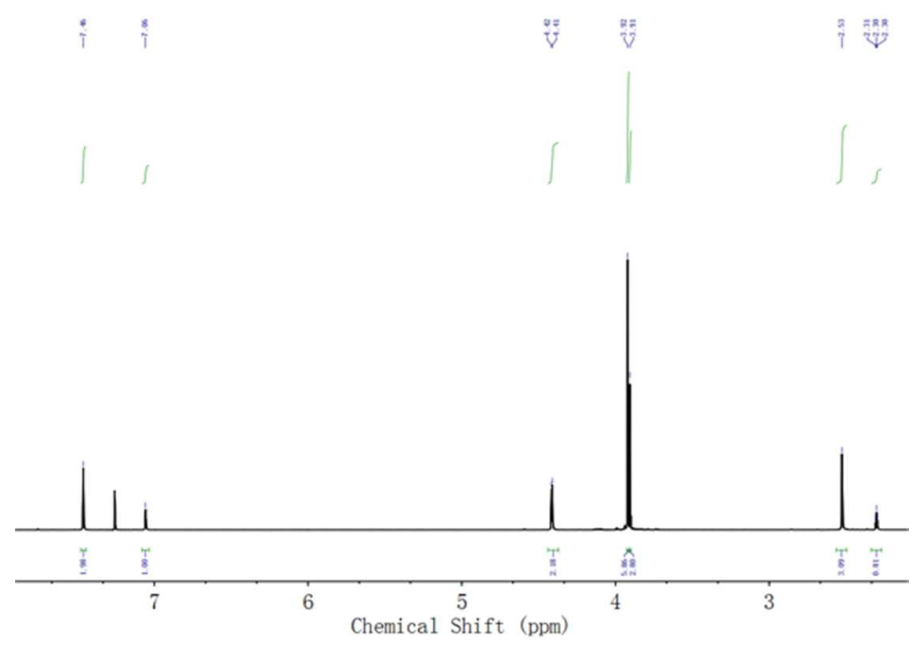

Figure S29. The ${ }^{1} \mathrm{H}$ NMR spectrum of 3,4,5-MeOBDPI. 


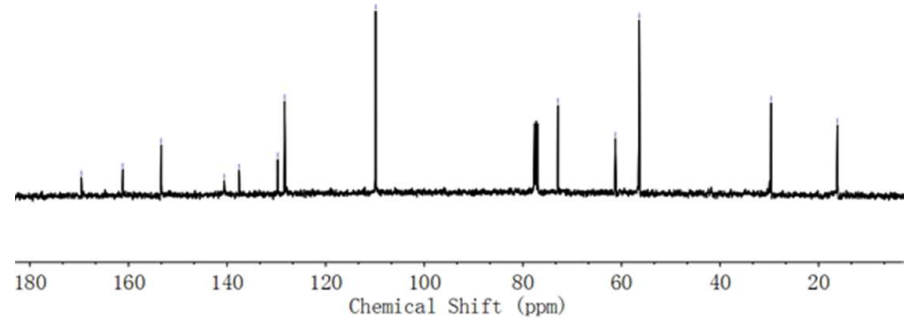

Figure S30. The ${ }^{13} \mathrm{C}$ NMR spectrum of $3,4,5$-MeOBDPI.

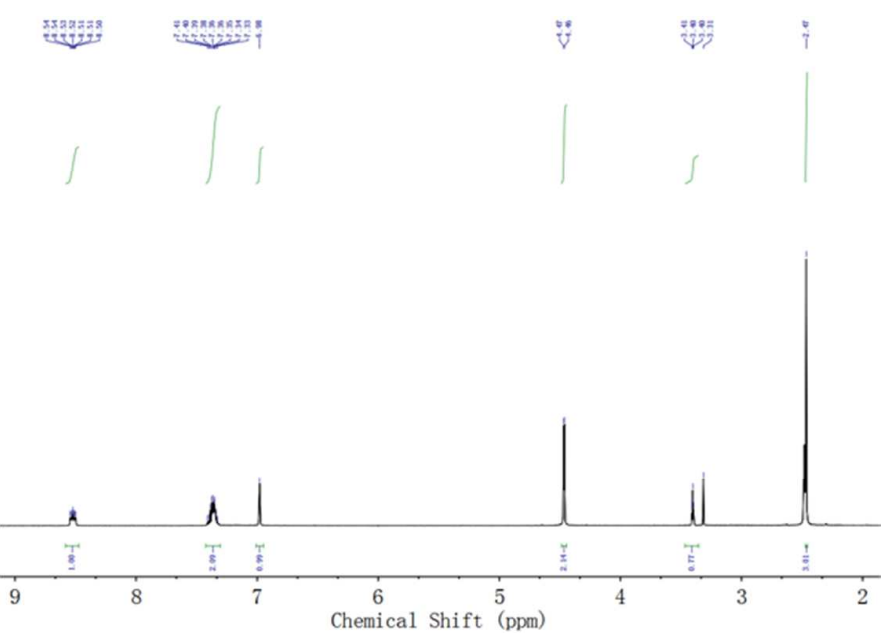

Figure S31. The ${ }^{1} \mathrm{H}$ NMR spectrum of 2,5-FBDPI.
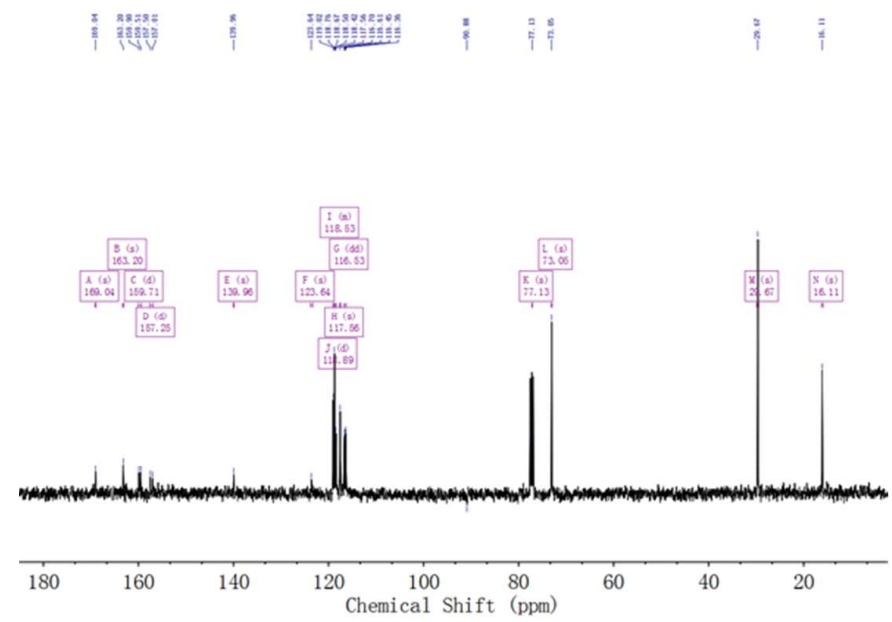

Figure S32. The ${ }^{13} \mathrm{C}$ NMR spectrum of 2,5-FBDPI. 


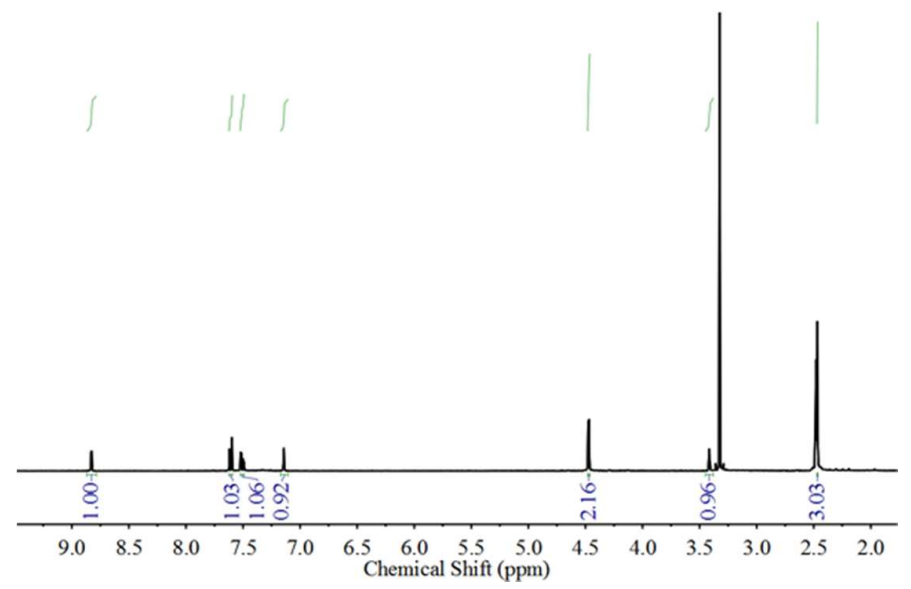

Figure S33. The ${ }^{1} \mathrm{H}$ NMR spectrum of 2,5-ClBDPI.
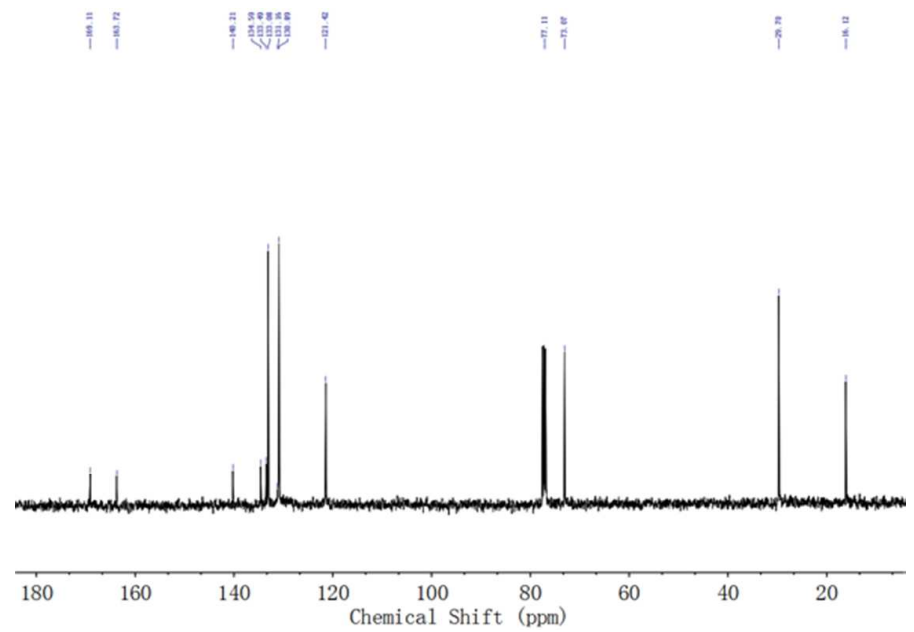

Figure S34. The ${ }^{13} \mathrm{C}$ NMR spectrum of 2,5-ClBDPI.

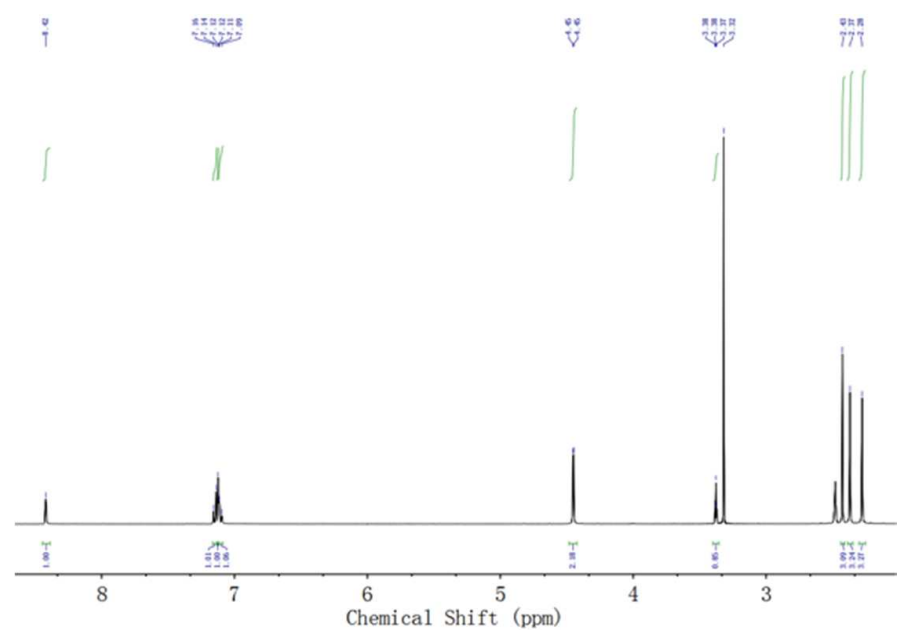

Figure S35. The ${ }^{1} \mathrm{H}$ NMR spectrum of 2,5-MeBDPI. 

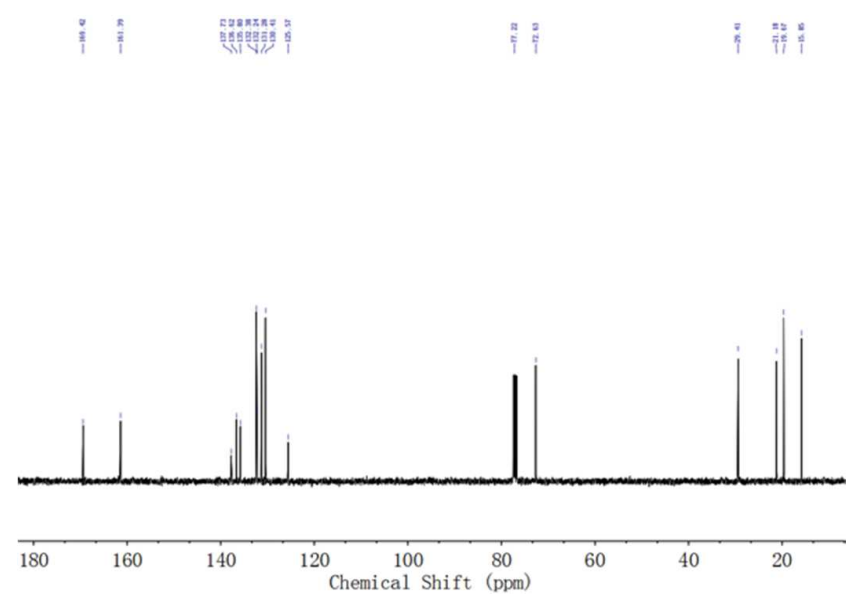

Figure S36. The ${ }^{13} \mathrm{C}$ NMR spectrum of 2,5-MeBDPI.

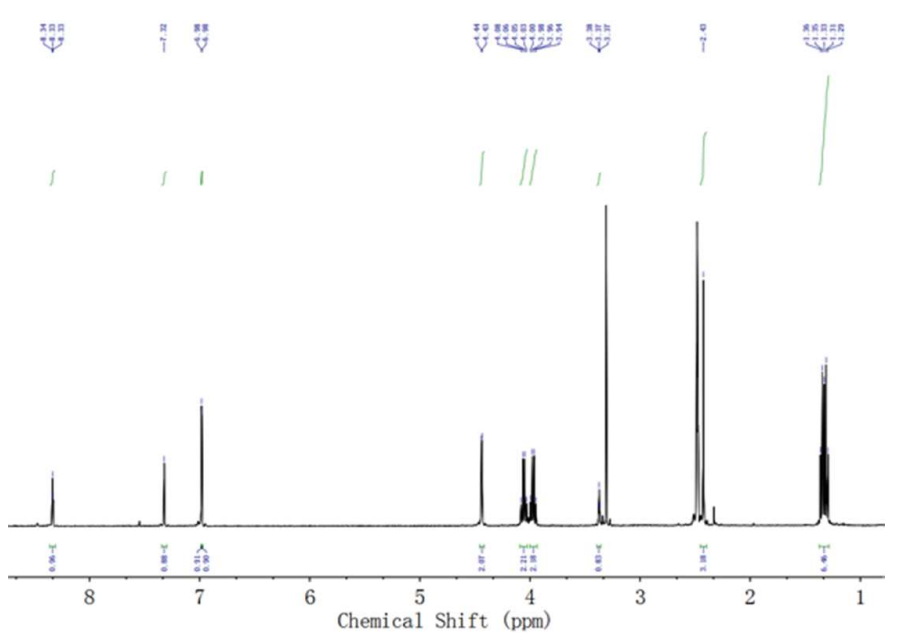

Figure S37. The ${ }^{1} \mathrm{H}$ NMR spectrum of 2,5-EtOBDPI.

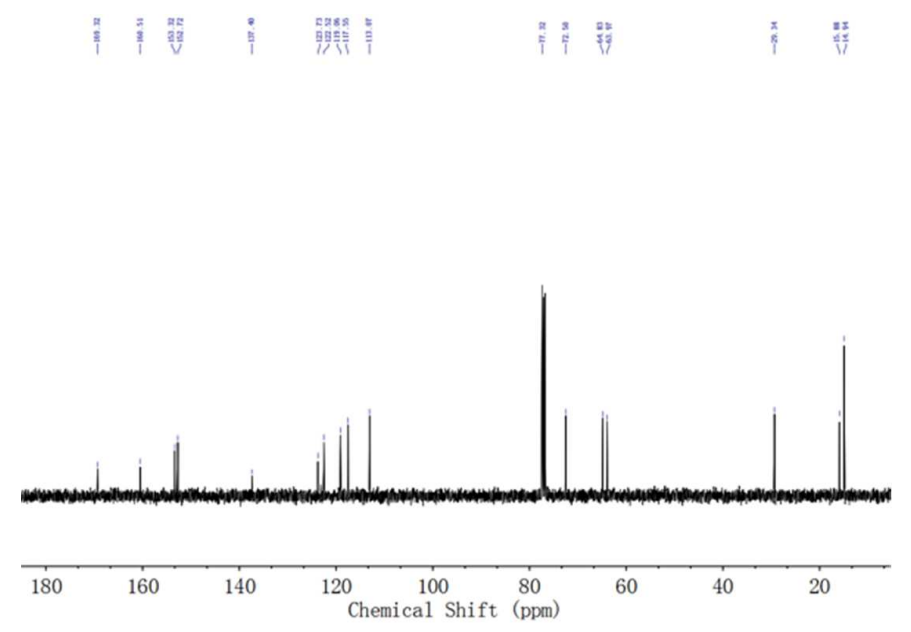

Figure S38. The ${ }^{13} \mathrm{C}$ NMR spectrum of 2,5-EtOBDPI. 


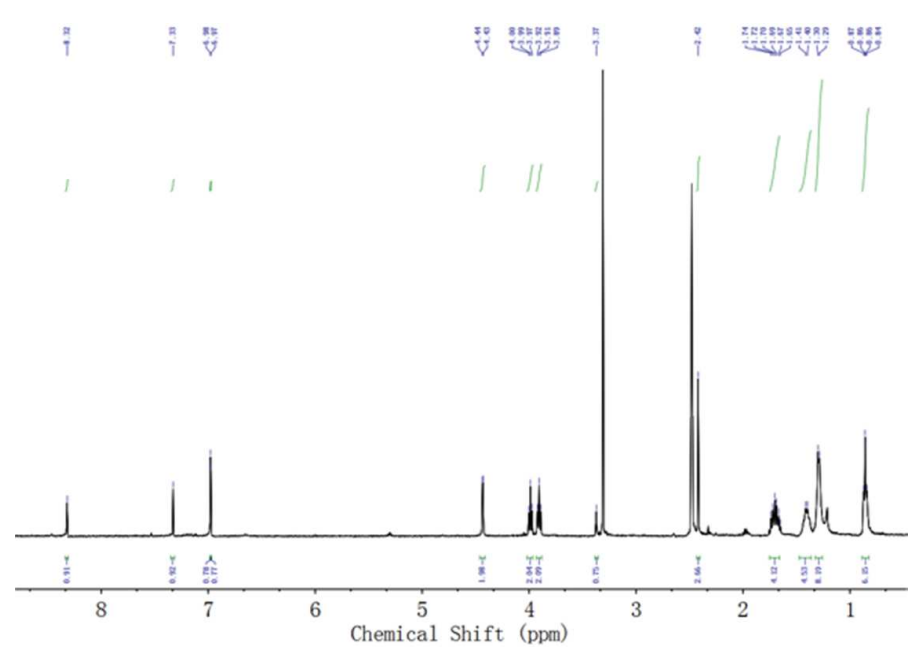

Figure S39. The ${ }^{1} \mathrm{H}$ NMR spectrum of 2,5-HexOBDPI.
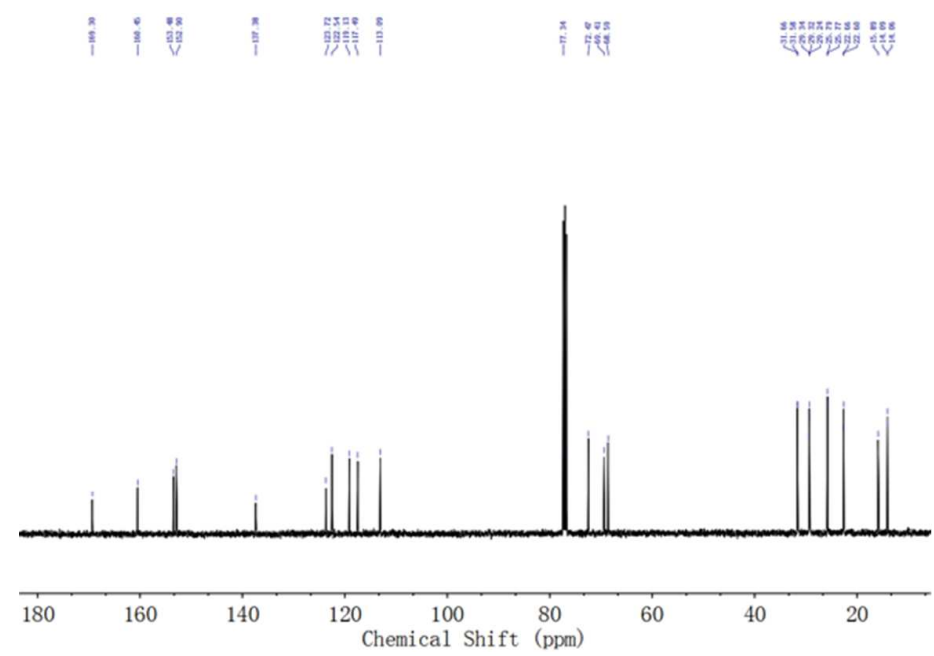

Figure S40. The ${ }^{13} \mathrm{C}$ NMR spectrum of 2,5-HexOBDPI.

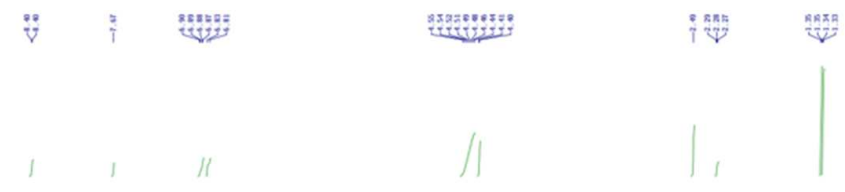

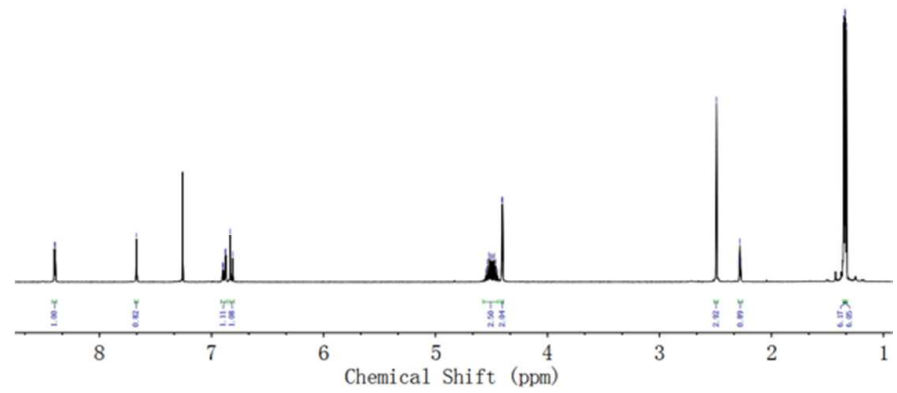

Figure S41. The ${ }^{1} \mathrm{H}$ NMR spectrum of 2,5-IprOBDPI. 


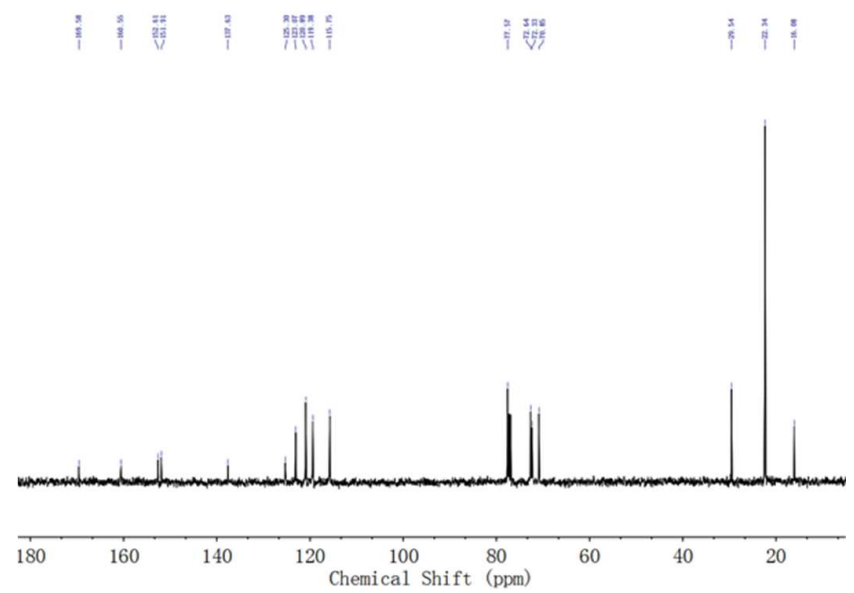

Figure S42. The ${ }^{13} \mathrm{C}$ NMR spectrum of 2,5-IprOBDPI. 
5. IR spectra for GFPc Analogs

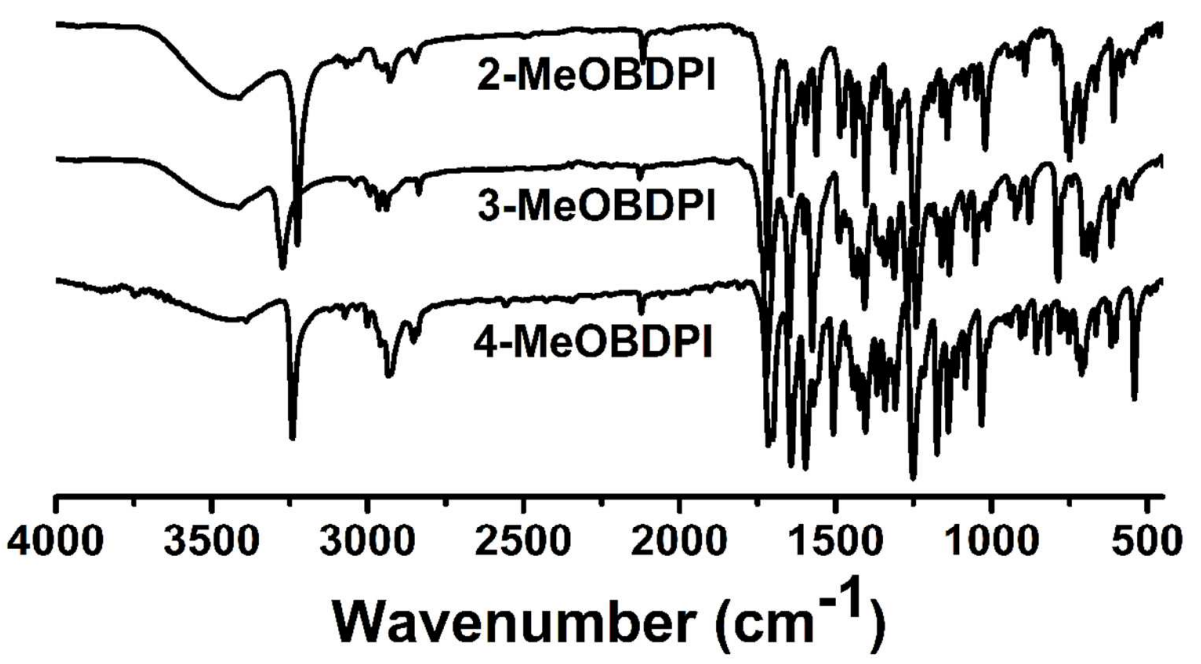

Figure S43. The IR spectra for 2-MeOBDPI, 3-MeOBDPI and 4-MeOBDPI.

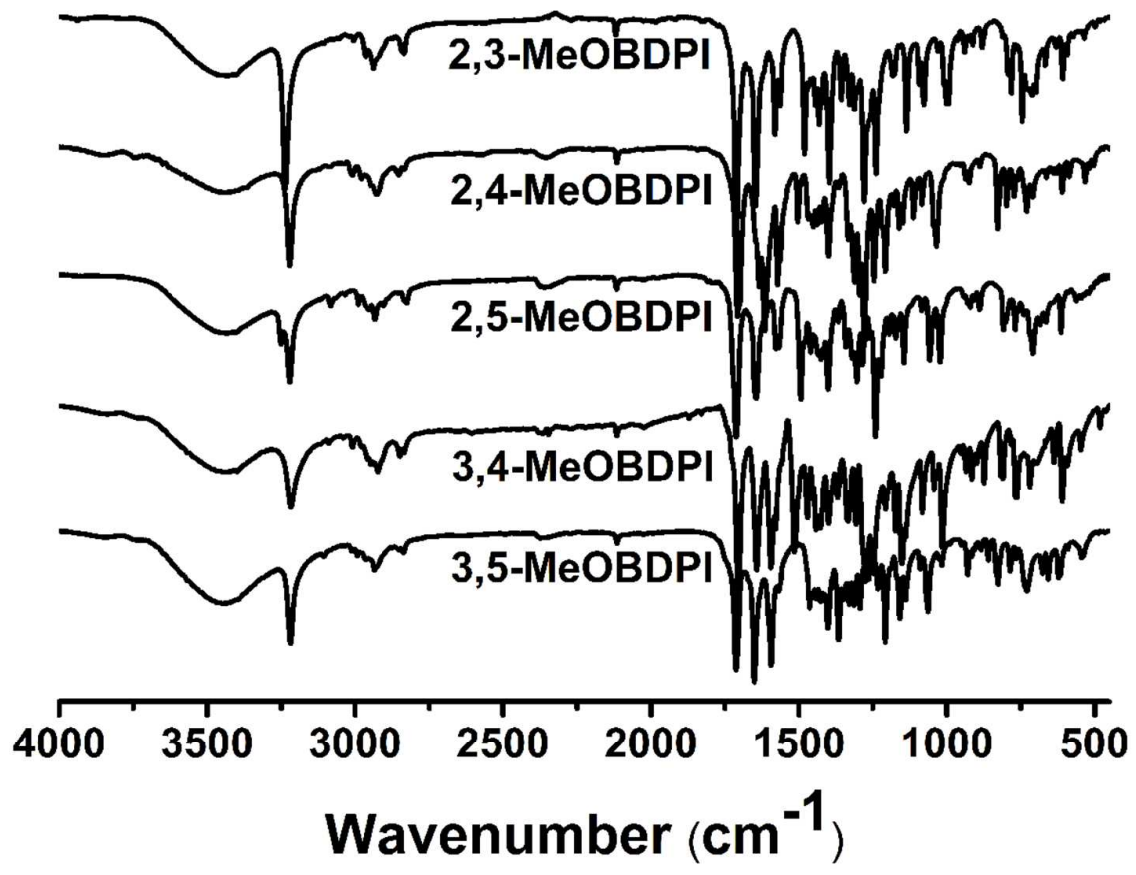

Figure S44. The IR spectra for 2,3-MeOBDPI, 2,4-MeOBDPI, 2,5-MeOBDPI, 3,4-MeOBDPI and 3,5-MeOBDPI. 


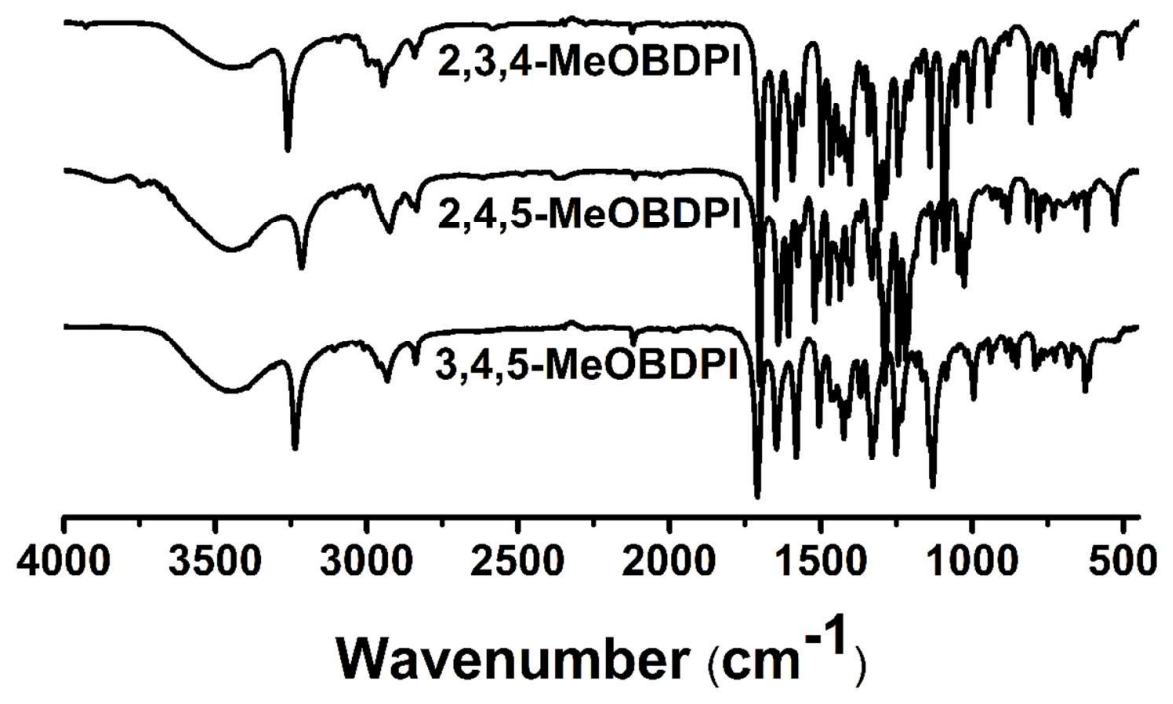

Figure S45. The IR spectra for 2,3,4-MeOBDPI, 2,4,5-MeOBDPI and 3,4,5-MeOBDPI.

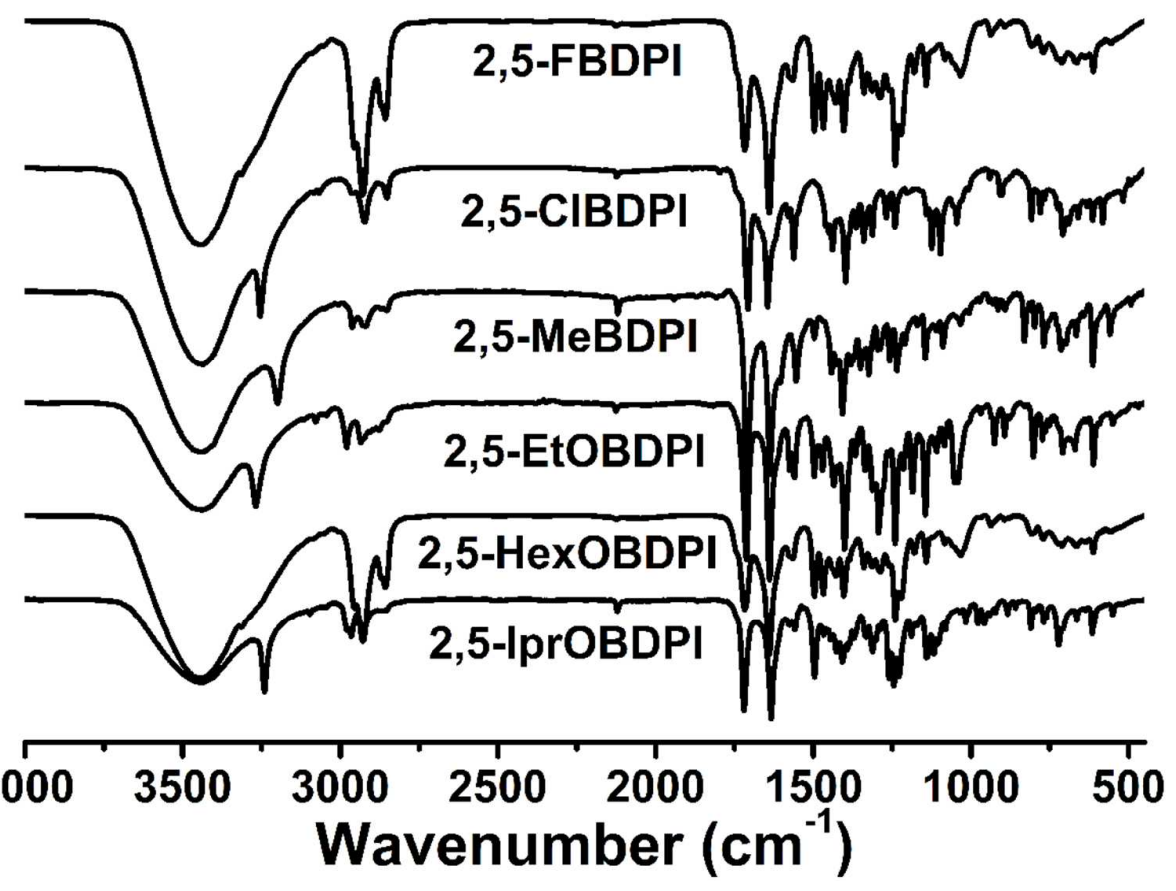

Figure S46. The IR spectra for 2,5-FBDPI, 2,5-ClBDPI, 2,5-MeBDPI, 2,5-EtOBDPI, 2,5-HexOBDPI and 2,5-IprOBDPI. 


\section{References}

(1) Baldridge, A.; Kowalik, J.; Tolbert, L. M. Efficient Synthesis of New 4-Arylideneimidazolin-5-ones Related to The GFP Chromophore by 2+3 Cyclocondensation of Arylideneimines with Imidate Ylides. Synthesis 2010, 14, 2424-2436.

(2) Sunahara, H.; Urano, Y.; Kojima, H.; Nagano, T. Design and Synthesis of A Library of BODIPY-Based Environmental Polarity Sensors Utilizing Photoinduced Electron-Transfer-Controlled Fluorescence ON/OFF Switching. J. Am. Chem. Soc. 2007, 129, 5597-5604.

(3) Frisch, M. J.; Trucks, G. W.; Schlegel, H. B.; Scuseria, G. E.; Robb, M. A.; Cheeseman, J. R.; Scalmani, G.; Barone, V.; Mennucci, B.; Petersson, G. A.; Nakatsuji, H.; Caricato, M.; Li, X.; Hratchian, H. P.; Izmaylov, A. F.; Bloino, J.; Zheng, G.; Sonnenberg, J. L.; Hada, M.; Ehara, M.; Toyota, K.; Fukuda, R.; Hasegawa, J.; Ishida, M.; Nakajima, T.; Honda, Y.; Kitao, O.; Nakai, H.; Vreven, T.; Montgomery, J. A.; Peralta, J. E.; Ogliaro, F.; Bearpark, M.; Heyd, J. J.; Brothers, E.; Kudin, K. N.; Staroverov, V. N.; Keith, T.; Kobayashi, R.; Normand, J.; Raghavachari, K.; Rendell, A.; Burant, J. C.; Iyengar, S. S.; Tomasi, J.; Cossi, M.; Rega, N.; Millam, J. M.; Klene, M.; Knox, J. E.; Cross, J. B.; Bakken, V.; Adamo, C.; Jaramillo, J.; Gomperts, R.; Stratmann, R. E.; Yazyev, O.; Austin, A. J.; Cammi, R.; Pomelli, C.; Ochterski, J. W.; Martin, R. L.; Morokuma, K.; Zakrzewski, V. G.; Voth, G. A.; Salvador, P.; Dannenberg, J. J.; Dapprich, S.; Daniels, A. D.; Farkas, O.; Foresman, J. B.; Ortiz, J. V.; Cioslowski, J.; Fox, D. J. Gaussian 09, revision D. 01; Gaussian, Inc. Wallingford CT, 2013.

(4) Vosko, S.; Wilk, L.; Nusair, M. Accurate Spin-Dependent Electron Liquid Correlation Energies for Local Spin Density Calculations: A Critical Analysis. Can. J. Phys. 1980, 58, 1200-1211.

(5) Becke, A. D. Density-Functional Thermochemistry. V. Systematic Optimization of Exchange-Correlation Functionals. J. Chem. Phys. 1997, 107, 8554-8560.

(6) Kohn, W.; Becke, A. D.; Parr, R. G. Density Functional Theory of Electronic Structure. J. Phys. Chem. 1996, 100, 12974-12980.

(7) Treutler, O.; Ahlrichs, R. Efficient Molecular Numerical Integration Schemes. J. Chem. Phys. 1995, 102, 346-354.

(8) Furche, F.; Ahlrichs, R. Adiabatic Time-Dependent Density Functional Methods for Excited State Properties. J. Chem. Phys. 2002, 117, 7433-7447. 